\title{
Inhomogeneous suspensions of rigid rods in flow
}

\author{
Jan K. G. Dhont ${ }^{\text {a) }}$ \\ Forschungszentrum Jülich, IFF/Weiche Materie, D-52425 Jülich, Germany \\ W. J. Briels ${ }^{\mathrm{b})}$ \\ Computational Dispersion Rheology, Department of Applied Physics, University of Twente, Postbus 217, \\ 7500 AE Enschede, The Netherlands
}

(Received 8 July 2002; accepted 21 October 2002)

\begin{abstract}
An expression for the divergence of the stress tensor is derived for inhomogeneous suspensions of very long and thin, rigid rods. The stress tensor is expressed in terms of the suspension flow velocity and the probability density function for the position and orientation of a rod. The expression for the stress tensor includes stresses arising from possibly very large spatial gradients in the shear rate, concentration, and orientational order parameter. The resulting Navier-Stokes equation couples to the equation of motion for the probability density function of the position and orientation of a rod. The equation of motion for this probability density function is derived from the $N$-particle Smoluchowski equation, including contributions from inhomogeneities. It is argued that for very long and thin rods, hydrodynamic interactions are of minor importance, and are therefore neglected, both in the expression for the stress tensor and in the equation of motion for the above-mentioned probability density function. The thus obtained complete set of equations of motion can be applied to describe phenomena where possibly very large spatial gradients occur, such as phase coexistence under shear flow conditions, including shear-banding, and phase separation kinetics. (C) 2003 American Institute of Physics. [DOI: 10.1063/1.1528912]
\end{abstract}

\section{INTRODUCTION}

In recent experiments on fd-virus suspensions under shear flow conditions, inside the two phase region for the isotropic-nematic phase transition, shear-banded stationary states are found at relatively low shear rates. ${ }^{1}$ The stationary state that is found is an alternating, banded structure that extends along the vorticity direction. In principle, stationary states also exist where coexistence of two regions occurs with differing shear rate, which extend in the gradient direction. These types of shear-banding are found in various systems which contain mesoscopic entities (see, e.g., Refs. $2-12$ ). In the nonequilibrium phase diagram, where the shear rate is one of the control variables, one should be able to mark regions where the various types of shear-banded structures are found. Furthermore, in regions where no shearbanding occurs, the location of paranematic-nematic spinodals and binodals will be shear-rate dependent. ${ }^{13}$ It is the aim of the present paper to derive, from microscopic considerations, a complete set of equations of motion for long and thin, rigid, hard rods, from which the nonequilibrium phase diagram can be calculated, including the shear-banding regions, and from which phase separation kinetics can be predicted. These equations of motion, in linearized form, should give insight into the microscopic origin of the shear-banding instability toward both types of above-mentioned banded structures. In particular, the origin of the instability that leads to banded structures that extend along the vorticity direction is not understood yet. Probably, the behavior of normal-stress differences drives this kind of instability. Phase separation

\footnotetext{
a)Electronic mail: j.k.g.dhont@fz-juelich.de

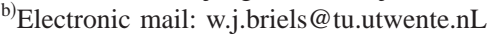

kinetics in regions in the phase diagram where shear-banding occurs is particularly interesting. Here two instabilities are at work simultaneously: the shear-banding instability and the instability that drives the isotropic-nematic phase transition. The interplay between these two instabilities (or metastabilities) determines the spatial morphologies in density and orientational order parameters during phase separation.

The only work that we are aware of that aims at a complete calculation of the nonequilibrium phase diagram, including shear-banding, is due to Olmsted. ${ }^{14-16}$ This is an admirable, pioneering piece of work, where a widely used approach is taken to obtain equations of motion. There are, however, a number of objections that can be raised to this approach. The probable reason for taking these objections for granted in literature is that, as yet, there is no general receipt to derive equations of motion for macroscopic quantities which apply to strongly inhomogeneous systems far away from equilibrium. First of all, the equations of motion that are used are partly based on thermodynamic arguments, where it is assumed that mass transport is driven by linear gradients in a chemical potential. This assumes (i) local equilibrium, (ii) the existence of a chemical potential, and (iii) small spatial gradients. Local equilibrium is questionable for strongly inhomogeneous systems far from global equilibrium. In some cases, however, one could argue that fast dynamics of small scale microstructure renders a system locally in equilibrium. Moreover, simple shear flow is a nonconservative external force field, for which no potential energy can be defined, and hence no free energy and chemical potential exist. This objection, however, may be academic in case there is local equilibrium. In that case the nonconservative nature of shear flow is mainly an issue for integrability of the 
equations of motion. Furthermore, large spatial gradients are generally present, which implies that higher order spatial gradients come into play in equations of motion. Even when local equilibrium can be assumed and the nonconservative nature of the shear field can be disregarded, the widely used approach referred to earlier probably fails in the presence of large spatial gradients. In the present paper we shall derive equations of motion from microscopic considerations, which do not rely on the above-mentioned assumptions. It is found that inhomogeneities are accounted for in the equations of motion by convolution-type integrals. Equations of motion that are similar (but not identical) in structure to those used by Olmsted are obtained after gradient expanding the convolution-type integrals, and truncating after the fourthorder derivative. These truncated equations of motion can be used only when spatial gradients are not very large. Such gradient expansions do not allow for the calculation of binodals, where indeed sharp interfaces occur, with very large spatial gradients. The equations of motion derived in the present paper allow one to assess the validity of gradient expanded equations of motion, and to calculate the entire phase diagram without having to rely on the abovementioned assumptions (this will be discussed in a future publication ${ }^{17}$ ).

In a previous paper ${ }^{18}$ we derived a general, microscopic expression for the divergence of the stress tensor for suspensions containing rigid colloidal particles. This result generalizes expressions of Batchelor ${ }^{19}$ and Doi and Edwards ${ }^{20}$ to include inhomogeneities in shear-rate, concentration, and orientational order parameters, and is valid for large spatial gradients in these suspension properties. In the present paper we shall evaluate this general expression for the stress tensor in case of very long and thin, rigid rods with excluded volume interactions. As it will turn out, the corresponding NavierStokes equation couples to the probability density function (pdf) of the position and orientation of a rod. An equation of motion is then derived for this pdf, starting from the $\mathrm{N}$-particle Smoluchowski equation. This equation of motion is also valid for large gradients of suspension properties. These two coupled, nonlinear equations of motion will be the starting point in a future publication to study phase behavior and phase separation kinetics under shear flow conditions, including shear-banding. ${ }^{17}$

In the derivation of the two above-mentioned equations of motion, we neglect hydrodynamic interactions between different rods. The reason for the probably small contributions of inter-rod hydrodynamic interactions (as compared to single particle contributions) is that the volume fraction $\varphi$ of interest scales like $\sim D / L$ for very long and thin rods (where $D$ is the thickness and $L$ the length of the rods). Due to the very small volume fractions of interest, hydrodynamic interactions are of minor importance, contrary to direct interactions. This issue will be discussed in some more detail in Sec. V.

This paper is organized as follows: In Sec. II we shall briefly discuss the general expression for the divergence of the stress tensor as derived in Ref. 18. Section III contains the derivation of the basic equations that are needed for the explicit evaluation of this general expression and the deriva- tion of the equation of motion for the probability density function (pdf) $\rho(\mathbf{r}, \hat{\mathbf{u}}, t)$ for the position $\mathbf{r}$ and orientation $\hat{\mathbf{u}}$ of a rod at time $t$. In Sec. IV, the various pdf's and ensemble averages are discussed which play a role in the evaluation of the divergence of the stress tensor in Sec. V. The derivation of the equation of motion for $\rho(\mathbf{r}, \hat{\mathbf{u}}, t)$ is presented in Sec. VI. Section VII is a summary and an outlook to the possible applications of the equations of motion.

\section{THE SUSPENSION STRESS TENSOR: GENERAL EXPRESSION}

Let us first briefly discuss the expression for the divergence of the suspension stress tensor $\Sigma(\mathbf{r}, t)$ as derived in Ref. 18. An expression for the body force $\nabla \cdot \Sigma(\mathbf{r}, t)$ at a point $\mathbf{r}$ in the suspension at time $t$ is obtained in Ref. 18 by simply adding the body forces that arise from (i) interactions between colloidal particles, (ii) interactions between colloidal particles and solvent molecules, and (iii) mutual interactions between solvent molecules. This reasoning is based on microscopic considerations, and does not rely on any thermodynamic arguments. For rigid, nondeformable colloidal particles, it is found that

$$
\begin{aligned}
\nabla \cdot \Sigma= & \eta_{0} \nabla^{2} \mathbf{U}(\mathbf{r}, t)-\nabla P^{s s}(\mathbf{r}, t) \\
& -\sum_{j=1}^{N}\left\langle\oint_{\partial V_{j}\left(\mathbf{r}_{j}, \hat{\mathbf{u}}_{j}\right)} d S^{\prime} \delta\left(\mathbf{r}-\mathbf{r}^{\prime}\right) \mathbf{f}^{h}\left(\mathbf{r}^{\prime}\right)\right\rangle,
\end{aligned}
$$

where $\eta_{0}$ is the shear viscosity of the incompressible solvent, $P^{s s}$ is the contribution to the suspension pressure due to interactions between solvent molecules, $\partial V_{j}\left(\mathbf{r}_{j}, \hat{\mathbf{u}}_{j}\right)$ is the surface area of $\operatorname{rod} j, \delta$ is the three-dimensional delta distribution, and $\mathbf{f}^{h}\left(\mathbf{r}^{\prime}\right)$ is the force per unit area that the solvent exerts on the surface area element at position $\mathbf{r}^{\prime}$ on the rods' surface (the superscript " $h$ " is used to indicate the hydrodynamic nature of this force). The first two terms on the righthand side arise from interactions between solvent molecules, while the last term is the combination of body forces arising from interactions of colloidal particles with other colloidal particles and solvent molecules. The angular brackets $\langle\cdots\rangle$ denote ensemble averaging with respect to the positions and orientations of the $N$ rigid colloidal particles in the system under consideration.

It should be noted that the hydrodynamic forces $\mathbf{f}^{h}$ in Eq. (1) implicitly depend on direct interactions between the rods. Direct interactions determine, to a large extent, the microstructural arrangement of the rods, which in turn affects hydrodynamic forces that are present between the surface elements of rods. This interplay between direct interactions and hydrodynamic interactions is, for example, illustrated by the force balance of hydrodynamic, direct and Brownian forces on the diffusive time scale, which has been used to derive Eq. (1) in Ref. 18.

The interpretation of Eq. (1) is relatively straightforward. The presence of the rigid colloidal particles contributes to the body force at a given point $\mathbf{r}$ in the system, due to the force $\mathbf{f}^{h}\left(\mathbf{r}^{\prime}\right)$ that a surface element on a colloidal particle at $\mathbf{r}^{\prime}$ exerts on the fluid. This force only contributes to the body 
force at $\mathbf{r}$ when the point $\mathbf{r}^{\prime}$ on the surface of a colloidal particle is located at $\mathbf{r}$, as signified by the delta distribution in the last term in Eq. (1).

In the derivation of the contribution to the stress tensor arising from interactions between solvent molecules [the first two terms on the right-hand side in Eq. (1)], it is assumed that contributions from microscopic mass-inhomogeneities can be neglected. The conditions under which this is valid are discussed in the appendix in Ref. 18, and are certainly satisfied for suspensions of very long and thin rods considered here. Volume fractions of interest (right up to the isotropic-nematic phase transition) are very small, which validates the neglect of microscopic mass-inhomogeneities.

Note that in the Navier-Stokes equation only the divergence of the stress tensor appears. In order to obtain an equation of motion for the suspension flow velocity, it is therefore sufficient to express the general expression (1) for the divergence of the stress tensor in terms of macroscopic quantities, like density and orientational order parameter.

The pressure $P^{s s}$ can be dealt with in two equivalent ways. One can either gradient expand the last term in Eq. (1), where terms of the form $\nabla f$ will be found, with $f$ a scalar field, and identify the total suspension pressure $P$ as $P^{s s}$ $+f$. Alternatively, $P^{s s}$ can be left as it stands, and be treated as an unknown scalar field, with incompressibility of the suspension flow $(\boldsymbol{\nabla} \cdot \mathbf{U}=0)$ added as an extra, independent field equation. We note here that $\nabla^{2} P \neq 0$, also for incompressible suspensions, since the suspension shear viscosity is not independent of position due to the presence of inhomogeneities. Hence, the total pressure (just like $P^{s s}$ ) depends not just on boundary conditions on the container walls, but also on the inhomogeneous morphology of the suspension. Just like the total suspension pressure $P$, also $P^{s s}$ depends on the shear-rate, concentration, and orientational order parameter(s) and their spatial gradients.

\section{SOME FUNDAMENTAL RELATIONS}

In order to explicitly evaluate Eq. (1) for the stress tensor in terms of concentration and the orientational order parameter, we shall need expressions which involve the hydrodynamic force distribution along the rod's long axis, and the translational and rotational velocity of a rod. These expressions should be valid for systems with large gradients in suspension properties.

For very long and thin rods, one may think of each rod as a rigid string of spherical beads with diameter $D$, as sketched in Fig. 1. There are $2 m+1=L / D$ beads, where $L$ is the total length and $D$ the diameter of a rod. The beads are indexed by the bead number $\alpha$, which ranges from $-m$ to $+m$. The bead with index $\alpha=0$ is located at the center $\mathbf{r}_{j}$ of the rod. The advantage of using a bead model for the rods is that it allows the use of Faxén's theorem for spherical objects (the beads) in order to evaluate hydrodynamic force distributions along the long axis of the rods. The bead model gives asymptotically correct results for very long and thin rods.

\section{A. Hydrodynamic forces on the beads of a rigid rod}

Consider a uniaxial rod of which the orientation is specified by the unit vector $\mathbf{u}$ pointing in the direction of the rod's

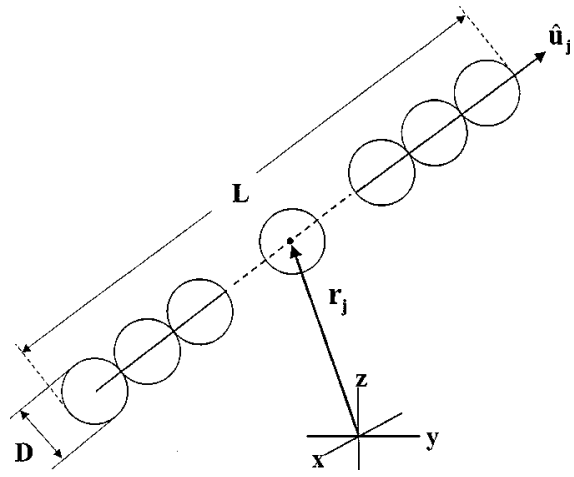

FIG. 1. The bead model for a rod. The spherical beads have a diameter $D$, and the total length is $L$. The aspect ratio is thus equal to $L / D=2 m+1$, where $2 m+1$ is the number of beads. The position coordinate $\mathbf{r}_{j}$ of the rod is the position of the center of mass, and the orientation is specified by the unit vector $\hat{\mathbf{u}}_{j}$ that points along the long axis of the rod.

long axis. Faxén's theorem for translational motion ${ }^{21,22}$ relates the force $\mathbf{F}_{\alpha}^{h}$ that the fluid exerts on bead $\alpha$ to the translational velocity $\mathbf{v}_{\alpha}$ of the bead and the fluid flow velocity $\mathbf{u}_{0, \alpha}$ that would have existed in the absence of bead $\alpha$, evaluated at the center of the bead (as indicated by the index " 0 "), as

$$
\mathbf{F}_{\alpha}^{h}=-\gamma \mathbf{v}_{\alpha}+\gamma\left[\mathbf{u}_{0, \alpha}+\frac{D^{2}}{24} \nabla_{\alpha}^{2} \mathbf{u}_{0, \alpha}\right],
$$

where $\gamma=3 \pi \eta_{0} D$ is the Stokes friction coefficient of a sphere with diameter $D$ in an unbounded fluid, and $\nabla_{\alpha}$ is the gradient operator with respect to the position coordinate of bead $\alpha$. The superscript " $h$ " is used to indicate the hydrodynamic nature of the force (other forces will be defined in Sec. III B). The first term in Eq. (2) describes the friction of the bead with the solvent as if it were in an unbounded, quiescent fluid, without the other beads being present. The second term is the hydrodynamic force due to hydrodynamic interactions with the other beads, belonging to the same rod and the other rods. The flow velocity $\mathbf{u}_{0, \alpha}$ is now written as a sum of two contributions, one stemming from the presence of the beads of the same rod, and a contribution arising from the presence of all other rods and a possible externally imposed flow field. The former contribution is related to the surface forces $\mathbf{f}_{\beta}^{h, \star}$ that the fluid exerts on surface elements of beads $\beta$ on the same rod to which bead $\alpha$ belongs, in case bead $\alpha$ would have been absent. The flow velocity $\mathbf{u}_{0, \alpha}$ is thus written as $^{21,22}$

$$
\mathbf{u}_{0, \alpha}=\mathbf{U}_{\alpha}^{\star}-\sum_{\beta \neq \alpha} \oint_{\partial V_{\beta}} d S^{\prime} \mathbf{T}\left(\mathbf{r}_{\alpha}-\mathbf{r}^{\prime}\right) \cdot \mathbf{f}_{\beta}^{h, \star}\left(\mathbf{r}^{\prime}\right),
$$

where the $\star$ 's indicate the absence of bead $\alpha$. Here, $\mathbf{U}_{\alpha}^{\star}$ is the fluid flow velocity at the position of bead $\alpha$ that is due to the presence of the remaining rods and the externally imposed flow field, in the absence of bead $\alpha$. It should be noted that the hydrodynamic forces $\mathbf{f}_{\beta}^{h, \star}$ are affected, through hydrodynamic interactions, by the presence of the other rods. Similarly, the field $\mathbf{U}_{\alpha}^{\star}$ is affected by the presence of the rod with the missing bead $\alpha$. Furthermore, $\mathbf{T}$ is the Oseen tensor, which is equal to ${ }^{21,22}$ 


$$
\mathbf{T}(\mathbf{r})=\frac{1}{8 \pi \eta_{0} r}[\hat{\mathbf{I}}+\hat{\mathbf{r}} \hat{\mathbf{r}}],
$$

where $\hat{\mathbf{I}}$ is the identity tensor and $\hat{\mathbf{r}}=\mathbf{r} / r$ the unit vector along r. For very long and thin rods, the difference between $\mathbf{f}_{\beta}^{h, \star}$ and the true surface force $\mathbf{f}_{\beta}^{h}$ on bead $\beta$, in the presence of bead $\alpha$, may be neglected. Only for a few neighboring beads to bead $\alpha$, the difference between the two forces may be significant, but for the majority of beads, further away from bead $\alpha$, the difference is insignificant. We shall henceforth replace $\mathbf{f}_{\beta}^{h, \star}$ by the true surface force $\mathbf{f}_{\beta}^{h}$. To within the bead model for the rod, for very long and thin rods, Eq. (3) then reduces to

$$
\mathbf{u}_{0, \alpha}=\mathbf{U}_{\alpha}^{\star}-\sum_{\beta \neq \alpha} \mathbf{T}\left(\mathbf{r}_{\alpha}-\mathbf{r}_{\beta}\right) \cdot \mathbf{F}_{\beta}^{h},
$$

where $\mathbf{F}_{\beta}^{h}$ is the hydrodynamic force on bead $\beta$,

$$
\mathbf{F}_{\beta}^{h}=\oint_{\partial V_{\beta}} d S^{\prime} \mathbf{f}^{h}\left(\mathbf{r}^{\prime}\right),
$$

with $\partial V_{\beta}$ the surface area of bead $\beta$. This expression can be substituted into Faxén's theorem (2). The term that involves the Laplacian is of higher order in $D / L$ relative to the other contributions, and will therefore be neglected in the following. Hence,

$$
\mathbf{F}_{j, \alpha}^{h}=-\gamma\left[\mathbf{v}_{j, \alpha}-\mathbf{U}_{j, \alpha}^{\star}\right]-\frac{3}{8}\left[\hat{\mathbf{I}}+\hat{\mathbf{u}}_{j} \hat{\mathbf{u}}_{j}\right] \cdot \sum_{\beta \neq \alpha} \frac{1}{|\alpha-\beta|} \mathbf{F}_{j, \beta}^{h},
$$

where we used that $\mathbf{r}_{j, \alpha}-\mathbf{r}_{j, \beta}=(\alpha-\beta) \hat{\mathbf{u}}_{j} D$, with $\hat{\mathbf{u}}_{j}$ the orientation of $\operatorname{rod} j$, which is the unit vector pointing along the long axis of the rod (see Fig. 1). Here, the rod number index $j$ is denoted explicitly for later reference: $\mathbf{v}_{j, \alpha}$ and $\mathbf{U}_{j, \alpha}^{\star}$ are $\mathbf{v}_{\alpha}$ and $\mathbf{U}_{\alpha}^{\star}$, where bead $\alpha$ belongs to rod $j$, respectively. This is a matrix equation for the bead force, which in principle can be solved once $\mathbf{v}_{j, \alpha}$ and $\mathbf{U}_{j, \alpha}^{\star}$ are specified.

On various occasions we shall need bead index summations of the form

$$
\sum_{\alpha} G(\alpha) \mathbf{F}_{j, \alpha}^{h},
$$

where $G$ is a well-behaved function of $\alpha$ that will be specified in each separate case in the sequel. Multiplying both sides of Eq. (7) with $G(\alpha)$ and summing over $\alpha$ leads to

$$
\begin{aligned}
\sum_{\alpha} G(\alpha) \mathbf{F}_{j, \alpha}^{h}= & -\gamma \sum_{\alpha} G(\alpha)\left[\mathbf{v}_{j, \alpha}-\mathbf{U}_{j, \alpha}^{\star}\right] \\
& -\frac{3}{8}\left[\hat{\mathbf{I}}+\hat{\mathbf{u}}_{j} \hat{\mathbf{u}}_{j}\right] \cdot \sum_{\alpha} \sum_{\beta \neq \alpha} \frac{G(\alpha)}{|\alpha-\beta|} \mathbf{F}_{j, \beta}^{h} .
\end{aligned}
$$

In Appendix $\mathrm{A}$ it is shown that, for the specific functions $G(\alpha)$ for which the bead index sum (8) is needed, this expression simplifies to

$$
\begin{aligned}
\sum_{\alpha} G(\alpha) \mathbf{F}_{j, \alpha}^{h}= & -\frac{4}{3} \frac{\gamma}{\ln \{L / D\}}\left[\hat{\mathbf{I}}-\frac{1}{2} \hat{\mathbf{u}}_{j} \hat{\mathbf{u}}_{j}\right] \\
& \cdot \sum_{\alpha} G(\alpha)\left[\mathbf{v}_{j, \alpha}-\mathbf{U}_{j, \alpha}^{\star}\right],
\end{aligned}
$$

for very long and thin rods. In this expression, terms that are of relative order $1 / \ln \{L / D\}$ are neglected. This result is crucial on various occasions in the sequel, in order to obtain an explicit expression for the divergence of the stress tensor and for the derivation of equations of motion for relevant probability density functions.

\section{B. The translational and rotational velocity of a rigid rod}

On the Brownian time scale, inertial forces are negligibly small in comparison to all other forces. ${ }^{22}$ The neglect of inertial forces implies a force balance between the other three forces acting on a rod: the force $\mathbf{F}_{j}^{h}$ that the fluid exerts on the rod (the superscript " $h$ " stands for "hydrodynamic"), the potential interaction force $\mathbf{F}_{j}^{I}=-\nabla_{j} \Psi$ (with $\nabla_{j}$ the gradient operator with respect to the position of rod number $j$ and $\Psi$ the total potential energy of the assembly of rods), and the Brownian force $\mathbf{F}_{j}^{\mathrm{Br}}=-k_{B} T \nabla_{j} \ln P$ (with $k_{B}$ Boltzmann's constant, $T$ the temperature, and $P$ the probability density function for the positions and orientations of all rods). Force balance implies that, on the Brownian time scale, these forces add up to $\mathbf{0}$ for each rod, and hence,

$$
\mathbf{F}_{j}^{h}=-\mathbf{F}_{j}^{I}-\mathbf{F}_{j}^{\mathrm{Br}}=\nabla_{j} \Psi+k_{B} T \nabla_{j} \ln P .
$$

The right-hand side is a function of positions and orientations. The total torque on a rod is analogously the sum of three contributions: the torque $\mathbf{T}_{j}^{h}$ that the fluid exerts on the rod, the potential interaction torque $\mathbf{T}_{j}^{I}=-\hat{\mathcal{R}}_{j} \Psi$, and the Brownian torque $\mathbf{T}_{j}^{\mathrm{Br}}=-k_{B} T \hat{\mathcal{R}}_{j} \ln P$. Here,

$$
\hat{\mathcal{R}}_{j} \equiv \hat{\mathbf{u}}_{j} \times \nabla_{\hat{\mathbf{u}}_{j}},
$$

is the rotation operator, with $\nabla_{\hat{\mathbf{u}}_{j}}$ the gradient operator with respect to the Cartesian coordinates of the orientation $\hat{\mathbf{u}}_{j}$ of $\operatorname{rod} j$. The rotational analog of Eq. (11) thus reads

$$
\mathbf{T}_{j}^{h}=-\mathbf{T}_{j}^{I}-\mathbf{T}_{j}^{\mathrm{Br}}=\hat{\mathcal{R}}_{j} \Psi+k_{B} T \hat{\mathcal{R}}_{j} \ln P .
$$

Now, $\mathbf{F}_{j}^{h}$ is given in Eq. (10) with $G(\alpha)=1$, since $\mathbf{F}_{j}^{h}$ $=\Sigma_{\alpha} \mathbf{F}_{j, \alpha}^{h}$ [that Eq. (10) is a good approximation for Eq. (9) with $G(\alpha)=1$ is shown in Appendix A]. Combining this with the force balance equation (11), and using that

$$
\mathbf{v}_{j, \alpha}=\mathbf{v}_{j}+\alpha D \boldsymbol{\Omega}_{j} \times \hat{\mathbf{u}}_{j},
$$

where $\mathbf{v}_{j}$ and $\boldsymbol{\Omega}_{j}$ are the translational and rotational velocity of rod $j$, respectively, it is readily found that

$$
\begin{aligned}
\nabla_{j} \Psi+k_{B} T \nabla_{j} \ln P= & -\frac{4}{3} \frac{\gamma}{\ln \{L / D\}} \frac{L}{D}\left[\hat{\mathbf{I}}-\frac{1}{2} \hat{\mathbf{u}}_{j} \hat{\mathbf{u}}_{j}\right] \\
& \cdot\left[\mathbf{v}_{j}-\frac{D}{L} \sum_{\alpha} \mathbf{U}_{j, \alpha}^{\star}\right] .
\end{aligned}
$$

The translational velocity of the $j$ th rod is thus found to be equal to

$$
\mathbf{v}_{j}=-D_{t} \mathbf{D}\left(\hat{\mathbf{u}}_{j}\right) \cdot\left[\beta \nabla_{j} \Psi+\nabla_{j} \ln P\right]+\frac{D}{L} \sum_{\alpha} \mathbf{U}_{j, \alpha}^{\star},
$$

with $\beta=1 / k_{B} T$ (not to be confused with a bead index number), and 


$$
D_{t}=\frac{k_{B} T \ln \{L / D\}}{4 \pi \eta_{0} L}
$$

is (proportional to) the translational diffusion coefficient for a free, noninteracting rod. ${ }^{22,23}$ Furthermore, the tensor,

$$
\mathbf{D}(\hat{\mathbf{u}}) \equiv \hat{\mathbf{I}}+\hat{\mathbf{u}} \mathbf{u},
$$

describes the orientational dependence of the translational friction coefficient of the rod.

The torque $\mathbf{T}_{j}^{h}$ that the fluid exerts on $\operatorname{rod} j$ is equal to

$$
\mathbf{T}_{j}^{h}=\oint_{\partial V_{j}\left(\mathbf{r}_{j}, \hat{\mathbf{u}}_{j}\right)} d S^{\prime}\left(\mathbf{r}^{\prime}-\mathbf{r}_{j}\right) \times \mathbf{f}^{h}\left(\mathbf{r}^{\prime}\right)=D \hat{\mathbf{u}}_{j} \times \sum_{\alpha} \alpha \mathbf{F}_{j, \alpha}^{h} .
$$

The sum $\Sigma_{\alpha} \alpha \mathbf{F}_{j, \alpha}^{h}$ is found from Eq. (10) with $G(\alpha)=\alpha$ [that Eq. (10) is a good approximation for Eq. (9) with $G(\alpha)=\alpha$ is shown in appendix A]. Hence,

$$
\begin{aligned}
\hat{\mathcal{R}}_{j} \Psi+k_{B} T \hat{\mathcal{R}}_{j} \ln P= & -\frac{\gamma D^{2}}{9 \ln \{L / D\}}\left(\frac{L}{D}\right)^{3} \\
& \times \hat{\mathbf{u}}_{j} \times\left[\Omega_{j} \times \hat{\mathbf{u}}_{j}-\frac{12}{D}\left(\frac{D}{L}\right)^{3} \sum_{\alpha} \alpha \mathbf{U}_{j, \alpha}^{\star}\right],
\end{aligned}
$$

where it is used that $\Sigma_{\alpha} \alpha^{2}=\frac{1}{12}(L / D)^{3}$ for long and thin rods (see Appendix A). Using that $\Omega_{j} \perp \hat{\mathbf{u}}_{j}$, it is found that,

$$
\Omega_{j}=-D_{r}\left[\beta \hat{\mathcal{R}}_{j} \Psi+\hat{\mathcal{R}}_{j} \ln P\right]+\frac{12}{D}\left(\frac{D}{L}\right)^{3} \hat{\mathbf{u}}_{j} \sum_{\alpha} \alpha \mathbf{U}_{j, \alpha}^{\star},
$$

where

$$
D_{r}=\frac{3 k_{B} T \ln \{L / D\}}{\pi \eta_{0} L^{3}}
$$

is the rotational diffusion coefficient for a free, noninteracting rod.

Note that in the above-given expressions, hydrodynamic interactions between rods are included through the solvent flow field $\mathbf{U}^{\star}$.

\section{THE RELEVANT PROBABILITY DENSITY FUNCTION AND THE PAIR-CORRELATION FUNCTION}

The divergence of the stress tensor in Eq. (1) will be expressed in terms of a probability density function (pdf). Furthermore, in the derivation of the equation of motion for this pdf in Sec. VI, the pair-correlation function is encountered. In the present section we shall define the relevant pdf and discuss a closure relation for the pair-correlation function.

The relevant pdf is that for the position $\mathbf{r}$ and orientation $\hat{\mathbf{u}}$ of a given rod, which is denoted as $P(\mathbf{r}, \hat{\mathbf{u}}, t)$. The number density $\rho(\mathbf{r}, \hat{\mathbf{u}}, t)$ at point $\mathbf{r}$ of rods having an orientation $\hat{\mathbf{u}}$ is equal to

$$
\rho(\mathbf{r}, \hat{\mathbf{u}}, t)=N P(\mathbf{r}, \hat{\mathbf{u}}, t),
$$

with $N$ the total number of rods in the system under consideration. Once this pdf is known, position-dependent orientational order parameters and concentrations can be calculated by integration.
Shear-rate-dependent locations of binodals for paranematic-nematic coexistence can be obtained by solving the equation of motion for $P(\mathbf{r}, \hat{\mathbf{u}}, t)$ [or, equivalently, $\rho(\mathbf{r}, \hat{\mathbf{u}}, t)]$ in conjunction with the Navier-Stokes equation, and then calculating by integration the orientational order parameters and density in the two bulk phases that coexist. In addition, the spatial dependence of these variables specifies the structure of the interface that connects the two bulk phases. It may happen that the stationary state is a shearbanded state rather then a state where two bulk phases coexist, in which case the position dependence of the density and the orientational order parameters describe the spatial structure of the shear-banded state.

The pdf $P(\mathbf{r}, \hat{\mathbf{u}}, t)$ is related to the pdf $P\left(\mathbf{r}_{1}, \ldots, \mathbf{r}_{N}, \hat{\mathbf{u}}_{1}, \ldots, \hat{\mathbf{u}}_{N}, t\right)$ of the phase space coordinates of all the $N$ rods in the system under consideration by

$$
\begin{aligned}
P\left(\mathbf{r}_{1}, \hat{\mathbf{u}}_{1}, t\right)= & \int d \mathbf{r}_{2} \cdots \int d \mathbf{r}_{N} \oint d \hat{\mathbf{u}}_{2} \cdots \oint d \hat{\mathbf{u}}_{N} \\
& \times P\left(\mathbf{r}_{1}, \mathbf{r}_{2}, \ldots, \mathbf{r}_{N}, \hat{\mathbf{u}}_{1}, \hat{\mathbf{u}}_{2}, \ldots, \hat{\mathbf{u}}_{N}, t\right),
\end{aligned}
$$

where the integrals $\oint$ range over all directions of $\hat{\mathbf{u}}$ 's, that is, over the entire unit spherical surface.

A second important pdf that is encountered in the explicit evaluation of Eq. (1) for the divergence of the stress tensor in Sec. V and the derivation of the equation of motion for $\rho(\mathbf{r}, \hat{\mathbf{u}}, t)$ in Sec. VI, is that for the positions and orientations of two rods, which is equal to

$$
\begin{aligned}
P\left(\mathbf{r}, \mathbf{r}^{\prime}, \hat{\mathbf{u}}, \hat{\mathbf{u}}^{\prime}, t\right)= & \int d \mathbf{r}_{3} \cdots \int d \mathbf{r}_{N} \oint d \hat{\mathbf{u}}_{3} \cdots \oint d \hat{\mathbf{u}}_{N} \\
& \times P\left(\mathbf{r}, \mathbf{r}^{\prime}, \mathbf{r}_{3}, \ldots, \mathbf{r}_{N}, \hat{\mathbf{u}}, \hat{\mathbf{u}}^{\prime}, \hat{\mathbf{u}}_{3}, \ldots, \hat{\mathbf{u}}_{N}, t\right) \\
= & P(\mathbf{r}, \hat{\mathbf{u}}, t) P\left(\mathbf{r}^{\prime}, \hat{\mathbf{u}}^{\prime}, t\right) g\left(\mathbf{r}, \mathbf{r}^{\prime}, \hat{\mathbf{u}}, \hat{\mathbf{u}}^{\prime}, t\right) \\
= & \frac{1}{N^{2}} \rho(\mathbf{r}, \hat{\mathbf{u}}, t) \rho\left(\mathbf{r}^{\prime}, \hat{\mathbf{u}}^{\prime}, t\right) g\left(\mathbf{r}, \mathbf{r}^{\prime}, \hat{\mathbf{u}}, \hat{\mathbf{u}}^{\prime}, t\right),
\end{aligned}
$$

where the second equation defines the pair-correlation function $g$. Note that the combination,

$$
\begin{aligned}
P\left(\mathbf{r}^{\prime}, \hat{\mathbf{u}}^{\prime} \mid \mathbf{r}, \hat{\mathbf{u}}, t\right) & \equiv P\left(\mathbf{r}, \mathbf{r}^{\prime}, \hat{\mathbf{u}}, \hat{\mathbf{u}}^{\prime}, t\right) / P(\mathbf{r}, \hat{\mathbf{u}}, t) \\
& =P\left(\mathbf{r}^{\prime}, \hat{\mathbf{u}}^{\prime}, t\right) g\left(\mathbf{r}, \mathbf{r}^{\prime}, \hat{\mathbf{u}}, \hat{\mathbf{u}}^{\prime}, t\right),
\end{aligned}
$$

is the conditional pdf of the position and orientation $\left\{\mathbf{r}^{\prime}, \hat{\mathbf{u}}^{\prime}\right\}$ of one rod for a prescribed position and orientation $\{\mathbf{r}, \hat{\mathbf{u}}\}$ of the second rod. We shall employ the low density approximation for the pair-correlation function in further derivations, that is,

$$
g\left(\mathbf{r}, \mathbf{r}^{\prime}, \hat{\mathbf{u}}, \hat{\mathbf{u}}^{\prime}, t\right)=\exp \left\{-\beta V\left(\mathbf{r}-\mathbf{r}^{\prime}, \hat{\mathbf{u}}, \hat{\mathbf{u}}^{\prime}\right)\right\} .
$$

Here, $V$ is the pair-interaction potential. Using arguments similar to that of Onsager, ${ }^{24}$ it is shown in Appendix B that Eq. (25) is a good approximation for the pair-correlation function for equilibrium systems of very long and thin rods with excluded volume interactions, provided that the degree of alignment is not too high. The approximation (25) is asymptotically correct in the limit where $D / L \rightarrow 0$, provided that the rods interact through short-ranged repulsive interactions and the suspension is in equilibrium. In using the form 
(25) in the present analysis we neglect nonequilibrium effects and effects of shear flow on $g$. Effects of shear flow are probably much less important than the shear aligning effects on the singlet pdf $P(\mathbf{r}, \hat{\mathbf{u}}, t)$.

Just as in the original equilibrium approach of Onsager, ${ }^{24}$ the validity of the approximation (25) for the paircorrelation function is valid in the isotropic state and the nematic state where the degree of alignment is to too high (such that the typical angle between two rods is larger than $D / L)$. Smectics cannot be described with the approximation (25). This limits the validity of the present work to concentrations where $(L / D) \varphi \leqslant 8$, approximately, where $\varphi$ is the volume fraction of rods. Furthermore, the form (25) for the pair-distribution function is not appropriate to describe systems of rods with attractive pair-interaction potentials. ${ }^{25}$ Here, we restrict ourselves to rods with excluded volume interactions only.

Note that for hard-core interactions we have the identity,

$$
\begin{aligned}
\exp \{ & \left.-\beta V\left(\mathbf{r}-\mathbf{r}^{\prime}, \hat{\mathbf{u}}, \hat{\mathbf{u}}^{\prime}\right)\right\} \nabla V\left(\mathbf{r}-\mathbf{r}^{\prime}, \hat{\mathbf{u}}, \hat{\mathbf{u}}^{\prime}\right) \\
& =-\beta^{-1} \nabla\left[\exp \left\{-\beta V\left(\mathbf{r}-\mathbf{r}^{\prime}, \hat{\mathbf{u}}^{\prime}, \hat{\mathbf{u}}^{\prime}\right)\right\}-1\right] \\
& =\beta^{-1} \nabla \chi\left(\mathbf{r}-\mathbf{r}^{\prime}, \hat{\mathbf{u}}, \hat{\mathbf{u}}^{\prime}\right),
\end{aligned}
$$

with $\chi$ the characteristic function of the excluded volume for two rods: $\chi=1$ when the cores of the two rods overlap and $\chi=0$ otherwise. The same relation holds when $\nabla$ is replaced by $\hat{\mathcal{R}}$.

\section{EXPLICIT EVALUATION OF THE STRESS TENSOR FOR VERY LONG AND THIN RODS}

For long and thin rods, the point $\mathbf{r}^{\prime}$ on the surface of the rod relative to the center-of-mass position of the rod can be written as $\mathbf{r}^{\prime}-\mathbf{r}_{j}=\alpha D \hat{\mathbf{u}}_{j}$. The surface integral that appears in Eq. (1) for the stress tensor can thus be written as a sum over beads as (with $\partial V_{\alpha}$ the surface area of bead $\alpha$ ),

$$
\begin{aligned}
& \oint_{\partial V_{j}\left(\mathbf{r}_{j}, \hat{\mathbf{u}}_{j}\right)} d S^{\prime} \delta\left(\mathbf{r}-\mathbf{r}^{\prime}\right) \mathbf{f}^{h}\left(\mathbf{r}^{\prime}\right) \\
& \quad=\sum_{\alpha} \delta\left(\mathbf{r}-\mathbf{r}_{j}-\alpha D \hat{\mathbf{u}}_{j}\right) \oint_{\partial V_{\alpha}} d S^{\prime} \mathbf{f}^{h}\left(\mathbf{r}^{\prime}\right) \\
& =\sum_{\alpha} \delta\left(\mathbf{r}-\mathbf{r}_{j}-\alpha D \hat{\mathbf{u}}_{j}\right) \mathbf{F}_{j, \alpha}^{h} .
\end{aligned}
$$

Hence,

$$
\begin{aligned}
\nabla \cdot \Sigma= & \eta_{0} \nabla^{2} \mathbf{U}(\mathbf{r}, \mathbf{t})-\nabla P^{s s}(\mathbf{r}, t) \\
& -\sum_{j=1}^{N} \sum_{\alpha}\left\langle\delta\left(\mathbf{r}-\mathbf{r}_{j}-\alpha D \hat{\mathbf{u}}_{j}\right) \mathbf{F}_{j, \alpha}^{h}\right\rangle .
\end{aligned}
$$

The sum with respect to $\alpha$ is of the form (8), where the ensemble averaging in Eq. (27) renders the function $G(\alpha)$ essentially equal to a probability density function, with the position of rod $j$ equal to $\mathbf{r}-\alpha D \hat{\mathbf{u}}_{j}$, as a result of the action of the delta distribution. Use of relation (10), whose validity in the present application is proven in Appendix A, immediately leads to

$$
\begin{aligned}
\nabla \cdot \Sigma= & \eta_{0} \nabla^{2} \mathbf{U}(\mathbf{r}, t)-\nabla P^{s s}(\mathbf{r}, t) \\
& +\frac{4}{3} \frac{\gamma}{\ln \{L / D\}}\left\langle\sum_{j=1}^{N}\left[\hat{\mathbf{I}}-\frac{1}{2} \hat{\mathbf{u}}_{j} \hat{\mathbf{u}}_{j}\right]\right. \\
& \left.\cdot \sum_{\alpha} \delta\left(\mathbf{r}-\mathbf{r}_{j}-\alpha D \hat{\mathbf{u}}_{j}\right)\left[\mathbf{v}_{j, \alpha}-\mathbf{U}_{j, \alpha}^{\star}\right]\right\rangle .
\end{aligned}
$$

For each $j$, the ensemble average appearing here that involves the solvent velocity $\mathbf{U}^{\star}$, can be written as

$$
\begin{gathered}
\left\langle\left[\hat{\mathbf{I}}-\frac{1}{2} \hat{\mathbf{u}}_{j} \hat{\mathbf{u}}_{j}\right] \cdot \sum_{\alpha} \delta\left(\mathbf{r}-\mathbf{r}_{j}-\alpha D \hat{\mathbf{u}}_{j}\right) \mathbf{U}_{j, \alpha}^{\star}\right\rangle \\
=\int d \mathbf{r}_{j} \oint d \hat{\mathbf{u}}_{j} P\left(\mathbf{r}_{j}, \hat{\mathbf{u}}_{j}, t\right)\left[\hat{\mathbf{I}}-\frac{1}{2} \hat{\mathbf{u}}_{j} \hat{\mathbf{u}}_{j}\right] \\
\cdot \sum_{\alpha} \delta\left(\mathbf{r}-\mathbf{r}_{j}-\alpha D \hat{\mathbf{u}}_{j}\right)\left\langle\mathbf{U}_{j, \alpha}^{\star}\right\rangle^{(c)},
\end{gathered}
$$

where $\langle\cdots\rangle^{(c)}$ denotes ensemble averaging with respect to the conditional pdf $P^{(c)}$ of $\left\{\mathbf{r}_{1}, \ldots, \mathbf{r}_{j-1}, \mathbf{r}_{j+1}, \ldots, \mathbf{r}_{N}\right.$, $\left.\hat{\mathbf{u}}_{1}, \ldots, \hat{\mathbf{u}}_{j-1}, \hat{\mathbf{u}}_{j+1}, \ldots, \hat{\mathbf{u}}_{N}\right\}$ for prescribed $\mathbf{r}_{j}$ and $\hat{\mathbf{u}}_{j}$, which is equal to

$$
\begin{aligned}
P^{(c)} & \left(\mathbf{r}_{1}, \ldots, \mathbf{r}_{j-1}, \mathbf{r}_{j+1}, \ldots, \mathbf{r}_{N},\right. \\
& \left.\times \hat{\mathbf{u}}_{1}, \ldots, \hat{\mathbf{u}}_{j-1}, \hat{\mathbf{u}}_{j+1}, \ldots, \hat{\mathbf{u}}_{N} \mid \mathbf{r}_{j}, \hat{\mathbf{u}}_{j}, t\right) \\
& \equiv P\left(\mathbf{r}_{1}, \ldots, \mathbf{r}_{N}, \hat{\mathbf{u}}_{1}, \ldots, \hat{\mathbf{u}}_{N}, t\right) / P\left(\mathbf{r}_{1}, \hat{\mathbf{u}}_{1}, t\right) .
\end{aligned}
$$

Using the definition in Eq. (21), we can thus rewrite the above-given expression for the divergence of the stress tensor as

$$
\begin{aligned}
\nabla \cdot \Sigma= & \eta_{0} \nabla^{2} \mathbf{U}(\mathbf{r}, t)-\nabla P^{s s}(\mathbf{r}, t) \\
& +\frac{4}{3} \frac{\gamma}{\ln \{L / D\}} \frac{1}{N} \sum_{j=1}^{N} \int d \mathbf{r}_{j} \oint d \hat{\mathbf{u}}_{j} \rho\left(\mathbf{r}_{j}, \hat{\mathbf{u}}_{j}, t\right) \\
& \times\left[\hat{\mathbf{I}}-\frac{1}{2} \hat{\mathbf{u}}_{j} \hat{\mathbf{u}}_{j}\right] \cdot \sum_{\alpha} \delta\left(\mathbf{r}-\mathbf{r}_{j}-\alpha D \hat{\mathbf{u}}_{j}\right) \\
& \times\left[\left\langle\mathbf{v}_{j, \alpha}\right\rangle^{(c)}-\left\langle\mathbf{U}_{j, \alpha}^{\star}\right\rangle^{(c)}\right] .
\end{aligned}
$$

The conditional ensemble average $\left\langle\mathbf{U}_{j, \alpha}^{\star}\right\rangle^{(c)}$ is the contribution to the solvent flow velocity at the position of bead $\alpha$ of $\operatorname{rod} j$, in the absence of that bead, that originates from the presence of other rods and the externally imposed flow, averaged over the positions and orientations of all other rods with a prescribed position and orientation of $\operatorname{rod} j$. We shall approximate this conditional average simply by the suspension flow velocity $\mathbf{U}_{j, \alpha}$ at the position of bead $\alpha$ of $\operatorname{rod} j$, that is,

$$
\left\langle\mathbf{U}_{j, \alpha}^{\star}\right\rangle^{(c)}=\mathbf{U}_{j, \alpha} .
$$

The argument for this approximation is as follows: The volume fractions of interest for very long and thin rods scale like $D / L,{ }^{24}$ and are therefore extremely small. As shown in Appendix C, this implies that the distance between beads on different rods, in terms of their diameter $D$, diverges in the limit where $L / D \rightarrow \infty$. Therefore, at some ratio $D / L$, hydrodynamic interactions should become unimportant relative to single particle contributions. As shown in Ref. 26, however, 
contributions to the stress tensor due to hydrodynamic interactions tend to 0 with decreasing $D / L$, relative to single particle contributions, very slowly. For experimental systems, hydrodynamic interaction contributions are of the order of $20 \%$ relative to single particle contributions. Hence, any numerical evaluation of the stress tensor based on the equations derived here is accurate up to about $20 \%$, relative to single particle contributions. Contributions due to direct interactions are much larger, as shown in Ref. 26 for homo- geneous systems. Incorporation of hydrodynamic interactions requires one to extend the above-given approximation with terms that include at least the leading contribution due to hydrodynamic interactions. This remains a future challenge.

Replacing $\mathbf{U}_{j, \alpha}^{\star}$ by $\mathbf{U}_{j, \alpha}$ after substitution of Eq. (14) for the velocity of bead $\alpha$ of $\operatorname{rod} j$, and subsequently using Eqs. (15) and (19) for the translational and rotational velocity, leads to an expression that can be written as (the nontrivial mathematical steps are illustrated in Appendix D)

$$
\begin{aligned}
\nabla \cdot \Sigma(\mathbf{r}, t)= & \eta_{0} \nabla^{2} \mathbf{U}(\mathbf{r}, t)-\nabla P^{s s}(\mathbf{r}, t)+\frac{k_{B} T}{L^{2}} \oint d \hat{\mathbf{u}} \int_{-L / 2}^{L / 2} d x\left\{12 \frac{x}{L} \hat{\mathbf{u}} \times\left[\hat{\mathcal{R}} \rho\left(\mathbf{r}-x \hat{\mathbf{u}}_{0}, \hat{\mathbf{u}}, t\right)\right] \hat{\mathbf{u}}_{0}=\hat{\mathbf{u}}-L \nabla \rho(\mathbf{r}-x \hat{\mathbf{u}}, \hat{\mathbf{u}}, t)\right\} \\
& +\frac{2 D k_{B} T}{L^{2}} \oint d \hat{\mathbf{u}} \oint d \hat{\mathbf{u}}^{\prime} \int_{-L / 2}^{L / 2} d x \int_{-L / 2}^{L / 2} d l \int_{-L / 2}^{L / 2} d l^{\prime} \rho(\mathbf{r}-x \hat{\mathbf{u}}, \hat{\mathbf{u}}, t) \\
& \times\left\{12 \frac{x}{L} \hat{\mathbf{u}} \times\left[\hat{\mathcal{R}}\left|\hat{\mathbf{u}} \times \hat{\mathbf{u}}^{\prime}\right| \rho\left(\mathbf{r}-x \hat{\mathbf{u}}_{0}-l \hat{\mathbf{u}}-l^{\prime} \hat{\mathbf{u}}^{\prime}, \hat{\mathbf{u}}^{\prime}, t\right)\right] \hat{\mathbf{u}}_{0}=\hat{\mathbf{u}}-\left|\hat{\mathbf{u}} \times \hat{\mathbf{u}}^{\prime}\right| L \nabla \rho\left(\mathbf{r}-(x+l) \hat{\mathbf{u}}-l^{\prime} \hat{\mathbf{u}}^{\prime}, \hat{\mathbf{u}}^{\prime}, t\right)\right\} \\
& +\frac{4 \pi \eta_{0}}{L \ln \{L / D\}} \oint d \hat{\mathbf{u}} \int_{-L / 2}^{L / 2} d x \int_{-L / 2}^{L / 2} d x^{\prime} \rho(\mathbf{r}-x \hat{\mathbf{u}}, \hat{\mathbf{u}}, t)\left[\hat{\mathbf{I}}-\frac{1}{2} \hat{\mathbf{u}} \hat{\mathbf{u}}\right] \\
& \cdot\left\{\mathbf{U}\left(\mathbf{r}+\left(x^{\prime}-x\right) \hat{\mathbf{u}}, t\right)-\mathbf{U}(\mathbf{r}, t)-12 \frac{x x^{\prime}}{L^{2}} \hat{\mathbf{u}} \times\left[\hat{\mathbf{u}} \times \mathbf{U}\left(\mathbf{r}+\left(x^{\prime}-x\right) \hat{\mathbf{u}}, t\right)\right]\right\} .
\end{aligned}
$$

Here, summations over bead indices $\alpha$ and $\beta$ are replaced by integrals with respect to $x$ and $x^{\prime}$, respectively [see Eqs. (D5) and (D8)]. The notation $[\hat{\mathcal{R}}(\cdots)]_{\hat{\mathbf{u}}_{0}=\hat{\mathbf{u}}}$ is used to indicate that the differentiation with respect to $\hat{\mathbf{u}}$ should be performed first, after which $\hat{\mathbf{u}}_{0}$ should be taken equal to $\hat{\mathbf{u}}$.

The first two contributions to the stress tensor are solvent contributions. The third term stems from Brownian forces, the fourth term from direct interactions, while the last term accounts for the suspension flow.

Contrary to commonly used expressions, Eq. (30) contains convolution-type integrals. An expression that is similar to commonly used expressions for the stress tensor is obtained by gradient expanding the convolution-type integrals and truncating this expansion after the fourth order in $\nabla$ contributions. Such a truncation is expected to work only when gradients are not very large. Our expression (30) for the divergence of the stress tensor, however, is valid even when large gradients are present in the system. In a future publication $^{17}$ we shall investigate the validity of gradient expansions by comparing paranematic-nematic interface profiles under shear flow as obtained from the gradient expanded version of Eq. (30) and its full convolution-type integral form [together with the corresponding equations of motion for the pdf $\rho(\mathbf{r}, \hat{\mathbf{u}}, t)]$.

Note that the central quantity in Eq. (30) is the joint probability density function $\rho(\mathbf{r}, \hat{\mathbf{u}}, t)$ for the position and orientation of a rod, and not the number density $\rho(\mathbf{r}, t)$ and the orientational order parameter tensor separately. The stress tensor can only be expressed in terms of density and order parameters in a truncated gradient expansion.

\section{EQUATION OF MOTION FOR $\rho(r, \hat{u}, t)$}

The Navier-Stokes equation contains the as yet unknown probability density function (pdf) $\rho(\mathbf{r}, \hat{\mathbf{u}}, t)$. We therefore need a separate equation that specifies that pdf. In the present section we shall derive an equation of motion for $\rho(\mathbf{r}, \hat{\mathbf{u}}, t)$ by integration of the Smoluchowski equation, which is the fundamental equation of motion for the $N$-particle pdf $P$. The Smoluchowski equation for rigid rods reads (for a derivation see, e.g., Ref. 22)

$$
\frac{\partial P}{\partial t}=-\sum_{j=1}^{N}\left[\nabla_{j} \cdot\left(\mathbf{v}_{j} P\right)+\hat{\mathcal{R}}_{j} \cdot\left(\mathbf{\Omega}_{j} P\right)\right],
$$

where, as before, $\mathbf{v}_{j}$ is the translational velocity of $\operatorname{rod} j$, and $\boldsymbol{\Omega}_{j}$ is it rotational velocity. Furthermore, $\nabla_{j}$ is the gradient operator with respect to the position coordinate $\mathbf{r}_{j}$ and $\hat{\mathcal{R}}_{j}$ is the rotational operator with respect to the orientation $\hat{\mathbf{u}}_{j}$. The rotational operator is defined in Eq. (12).

The equation of motion of interest here is the equation of motion for the number density $\rho(\mathbf{r}, \hat{\mathbf{u}}, t)$, as defined in sec. IV. According to Eq. (22), this equation of motion is obtained by integration of the Smoluchowski equation with respect to $\mathbf{r}_{2}, \ldots, \mathbf{r}_{N}$ and $\hat{\mathbf{u}}_{2}, \ldots, \hat{\mathbf{u}}_{N}$. According to the integral theorems of Gauss and Stokes we have 


$$
\begin{aligned}
& \int d \mathbf{r}_{j} \nabla_{j} \cdot(\cdots)=0, \\
& \oint d \hat{\mathbf{u}}_{j} \hat{\mathcal{R}}_{j} \cdot(\cdots)=\oint d \hat{\mathbf{u}}_{j} \hat{\mathbf{u}}_{j} \cdot \nabla_{\mathbf{u}_{j}} \times(\cdots)=0,
\end{aligned}
$$

where the integrals $\oint$ are surface integrals extending over the unit spherical surface. Hence, upon integration with respect to $\left\{\mathbf{r}_{2}, \ldots, \mathbf{r}_{N}\right\}$ and $\left\{\hat{\mathbf{u}}_{2}, \ldots, \hat{\mathbf{u}}_{N}\right\}$, only the terms with $j=1$ in the Smoluchowski equation (31) survive. For a pairwise additive total potential [see Eq. (D6)], it is found from Eqs. (15), (19), (29) and (31) that for identical rods (with $\mathbf{r}=\mathbf{r}_{1}$, $\hat{\mathbf{u}}=\hat{\mathbf{u}}_{1}$, and $\left.\mathbf{U}_{\alpha}=\mathbf{U}_{1, \alpha}\right)$,

$$
\begin{aligned}
\frac{\partial}{\partial t} \rho(\mathbf{r}, \hat{\mathbf{u}}, t)= & D_{t} \nabla \cdot \mathbf{D}(\hat{\mathbf{u}}) \cdot\{\nabla \rho(\mathbf{r}, \hat{\mathbf{u}} ; t)-\beta \rho(\mathbf{r}, \hat{\mathbf{u}}, t) \overline{\mathbf{F}}(\mathbf{r}, \hat{\mathbf{u}}, t)\} \\
& +D_{r} \hat{\mathcal{R}} \cdot\{\hat{\mathcal{R}} \rho(\mathbf{r}, \hat{\mathbf{u}}, t)-\beta \rho(\mathbf{r}, \hat{\mathbf{u}}, t) \overline{\mathbf{T}}(\mathbf{r}, \hat{\mathbf{u}}, t)\} \\
& -\nabla \cdot \rho(\mathbf{r}, \hat{\mathbf{u}}, t) \frac{1}{L} \int_{-L / 2}^{L / 2} d x \mathbf{U}(\mathbf{r}+x \hat{\mathbf{u}}, t) \\
& -\hat{\mathcal{R}} \cdot \rho(\mathbf{r}, \hat{\mathbf{u}}, t) \frac{12}{L^{3}} \hat{\mathbf{u}} \times \int_{-L / 2}^{L / 2} d x x \mathbf{U}(\mathbf{r}+x \hat{\mathbf{u}}, t),
\end{aligned}
$$

with $\nabla$ the gradient operator with respect to $\mathbf{r}$ and $\hat{\mathcal{R}}$ the rotational operator with respect to $\hat{\text { u. }}$ Here, we replaced bead index summations by integrals [see Eqs. (D5) and (D8)]. The average force $\overline{\mathbf{F}}$ and torque $\overline{\mathbf{T}}$ are equal to (with $\mathbf{r}^{\prime}=\mathbf{r}_{2}$ and $\left.\hat{\mathbf{u}}^{\prime}=\hat{\mathbf{u}}_{2}\right)$

$$
\begin{aligned}
\overline{\mathbf{F}}(\mathbf{r}, \hat{\mathbf{u}}, t)= & -\int d \mathbf{r}^{\prime} \oint d \hat{\mathbf{u}}^{\prime} \rho\left(\mathbf{r}^{\prime}, \hat{\mathbf{u}}^{\prime}, t\right) g\left(\mathbf{r}, \mathbf{r}^{\prime}, \hat{\mathbf{u}}, \hat{\mathbf{u}}^{\prime}, t\right) \\
& \times \nabla V\left(\mathbf{r}-\mathbf{r}^{\prime}, \hat{\mathbf{u}}, \hat{\mathbf{u}}^{\prime}\right),
\end{aligned}
$$

and

$$
\begin{aligned}
\overline{\mathbf{T}}(\mathbf{r}, \hat{\mathbf{u}}, t)= & -\int d \mathbf{r}^{\prime} \oint d \hat{\mathbf{u}}^{\prime} \rho\left(\mathbf{r}^{\prime}, \hat{\mathbf{u}}^{\prime}, t\right) g\left(\mathbf{r}, \mathbf{r}^{\prime}, \hat{\mathbf{u}}, \hat{\mathbf{u}}^{\prime}, t\right) \\
& \times \hat{\mathcal{R}} V\left(\mathbf{r}-\mathbf{r}^{\prime}, \hat{\mathbf{u}}, \hat{\mathbf{u}}^{\prime}\right),
\end{aligned}
$$

where the pair-correlation function $g$ is defined in Eq. (23). Note that according to Eq. (24) for the conditional pdf, these are the force and torque on a rod with prescribed orientation $\hat{\mathbf{u}}$ and position $\mathbf{r}$, averaged over the orientations and positions of other rods. The average is thus with respect to the conditional pdf for $\left\{\mathbf{r}^{\prime}, \hat{\mathbf{u}}^{\prime}\right\}$, given that the rod under consideration is at $\{\mathbf{r}, \hat{\mathbf{u}}\}$. Using Eq. (26) for the pair-correlation function, the force (34) and torque (35) are found to be equal to

$$
\overline{\mathbf{F}}(\mathbf{r}, \hat{\mathbf{u}}, t)=-\nabla V^{\mathrm{eff}}(\mathbf{r}, \hat{\mathbf{u}}, t), \quad \overline{\mathbf{T}}(\mathbf{r}, \hat{\mathbf{u}}, t)=-\hat{\mathcal{R}} V^{\mathrm{eff}}(\mathbf{r}, \hat{\mathbf{u}}, t),
$$

where the effective potential $V^{\text {eff }}$ is equal to (with $\left.\mathbf{R}=\mathbf{r}^{\prime}-\mathbf{r}\right)$,

$$
V^{\mathrm{eff}}(\mathbf{r}, \hat{\mathbf{u}}, t)=\beta^{-1} \int d \mathbf{R} \oint d \hat{\mathbf{u}}^{\prime} \rho\left(\mathbf{r}+\mathbf{R}, \hat{\mathbf{u}}^{\prime}, t\right) \chi\left(\mathbf{R}, \hat{\mathbf{u}}, \hat{\mathbf{u}}^{\prime}\right) .
$$

Since suspension properties are constant over distances of the order of the thickness $D$ of the rods, the effective potential (37) can be written in a convolution-type integral form as

$$
\begin{aligned}
V^{\mathrm{eff}}(\mathbf{r}, \hat{\mathbf{u}}, t)= & \frac{1}{2} D L^{2} \beta^{-1} \oint d \hat{\mathbf{u}}^{\prime}\left|\hat{\mathbf{u}} \times \hat{\mathbf{u}}^{\prime}\right| \int_{-1}^{+1} d l \int_{-1}^{+1} d l^{\prime} \\
& \times \rho\left(\mathbf{r}+\frac{1}{2} L l \hat{\mathbf{u}}+\frac{1}{2} L l^{\prime} \hat{\mathbf{u}}^{\prime}, \hat{\mathbf{u}}^{\prime}, t\right) .
\end{aligned}
$$

Note that for a homogeneous system, where $\rho\left(\mathbf{r}, \hat{\mathbf{u}}^{\prime}, t\right)$ $=\bar{\rho} P\left(\hat{\mathbf{u}}^{\prime}, t\right) \quad($ with $\bar{\rho}=N / V$ the overall number density of rods), the integral in Eq. (37) with respect to $\mathbf{R}$ yields the overlap volume for two rods, so that

$$
V^{\mathrm{eff}}(\hat{\mathbf{u}}, t)=2 D L^{2} \bar{\rho} \beta^{-1} \oint d \hat{\mathbf{u}}^{\prime}\left|\hat{\mathbf{u}} \times \hat{\mathbf{u}}^{\prime}\right| P\left(\hat{\mathbf{u}}^{\prime}, t\right) .
$$

This is the well-known Doi-Edwards potential for homogeneous suspensions. ${ }^{20}$

To within a Ginzburg-Landau expansion up to third order in the orientational order parameter (which amounts to using the Maier-Saupe potential) and subsequently gradient expansion of Eq. (37) to leading order in gradients, leads to an expression for $V^{\text {eff }}$ that can be compared to an expression for the effective potential as proposed by Greco and Marrucci (see Ref. 27, and references therein). A quite different expression is found as compared to that of Greco and Marrucci. The Laplacian of the orientational order parameter in the Greco-Marrucci expression is not reproduced, and a number of quite different gradient terms are found to contribute to $V^{\text {eff }}$.

Equations (33), (36) and (38) specify the equation of motion for $\rho(\mathbf{r}, \hat{\mathbf{u}}, t)$. As mentioned before, the NavierStokes equation couples to this equation of motion through Eq. (30) for the divergence of the stress tensor that contains the density $\rho(\mathbf{r}, \hat{\mathbf{u}}, t)$, while the above-mentioned Eq. (33) couples to the Navier-Stokes equation through its dependence on the suspension velocity. We thus have a closed set of two, nonlinearly coupled equations of motion which describe nonequilibrium phenomena and stationary states under shear flow, where large spatial gradients may play a crucial role.

\section{SUMMARY AND CONCLUSIONS}

A microscopic approach for the derivation of the Navier-Stokes equation for possibly very inhomogeneous systems of long and thin, hard and rigid rods is described. The Navier-Stokes equation couples to the equation of motion for the probability density function for the position and orientation of a rod. The equation of motion for this quantity is derived, again within a microscopic approach. This renders a closed set of equations which allows for the analysis of phase behavior and phase separation kinetics under shear flow conditions, including shear-banding. Since the equations of motion are valid for possibly large gradients in shear-rate, concentration, and orientational order parameters, these equations can be used to describe phase separation also in the late stages and to obtain the shear-rate-dependent location of binodals, where sharp interfaces must be correctly accounted for. Inhomogeneities are accounted for by 
convolution-type integrals. Upon gradient expanding these integrals up to fourth-order terms, one should recover equations of motion that are similar to those used in previous studies. Our results thus allow one to assess the validity of such gradient expanded equations of motion (in fact, binodals cannot be calculated on the basis of such truncated gradient expansions). The approximations underlying the equations of motion are that (i) the pair-correlation function is simply the Boltzmann exponential of the pair-interaction potential and (ii) hydrodynamic interactions between different rods are neglected. The first approximation is exact for very long and thin rods in equilibrium and is probably a good approximation for nonequilibrium systems under shear flow, while the validity of the second approximation is related to the very low volume fractions of interest for the very long rods under consideration. The Navier-Stokes equation with a body force given in Eq. (30) and the equation of motion (33) will be analyzed in future publications.

\section{ACKNOWLEDGMENT}

W.J.B.'s contribution is part of the research program of the "Stichting voor Fundamenteel Onderzoek der Materie (FOM)," which is financially supported by the "Nederlandse Organisatie voor Wetenschappelijk Onderzoek (NWO)."

\section{APPENDIX A: EVALUATION OF SUMS OVER BEAD INDEX NUMBERS}

The simplest summations with respect to bead indices encountered in the main text are of the form, $\Sigma_{\alpha} \alpha^{n}$, with $n$ a positive integer. The summation ranges from $\alpha=-m$ to $m$, with $2 m+1=L / D$. For large aspect ratio this summation can be replaced by an integral. To leading order in $L / D$ this leads to,

$$
\begin{aligned}
\sum_{\alpha} \alpha^{n} & =\int_{-(1 / 2)(L / D-1)}^{(1 / 2)(L / D-1)} d x x^{n} \\
& =\frac{2^{-n}}{n+1}\left(\frac{L}{D}\right)^{n+1}, \quad \text { for even } n .
\end{aligned}
$$

This sum is 0 for odd $n$. In particular, we have

$$
\sum_{\alpha} \alpha^{2}=\frac{1}{12}\left(\frac{L}{D}\right)^{3}, \quad \sum_{\alpha} \alpha^{4}=\frac{1}{80}\left(\frac{L}{D}\right)^{3} .
$$

Much more complicated is the approximate evaluation of Eq. (9) to obtain an explicit expression for the sum in Eq. (8). As a first step, the double sum in Eq. (9) is rewritten as

$$
\begin{aligned}
\sum_{\alpha} \sum_{\beta \neq \alpha} \frac{G(\alpha)}{|\alpha-\beta|} \mathbf{F}_{j, \beta}^{h}= & \sum_{\alpha} \sum_{\beta \neq \alpha} \frac{G(\beta)}{|\alpha-\beta|} \mathbf{F}_{j, \beta}^{h} \\
& +\sum_{\alpha} \sum_{\beta \neq \alpha} \frac{G(\alpha)-G(\beta)}{|\alpha-\beta|} \mathbf{F}_{j, \beta}^{h} .
\end{aligned}
$$

The last term in Eq. (A2) can be rewritten, by first interchanging the summation indices $\alpha$ and $\beta$, and subsequently interchanging the order of summations, as

$$
\begin{aligned}
\sum_{\alpha} & \sum_{\beta \neq \alpha} \frac{G(\alpha)-G(\beta)}{|\alpha-\beta|} \mathbf{F}_{j, \beta}^{h} \\
& =\sum_{\beta} \sum_{\alpha \neq \beta} \frac{G(\beta)-G(\alpha)}{|\alpha-\beta|} \mathbf{F}_{j, \alpha}^{h} \\
& =\sum_{\alpha} \mathbf{F}_{j, \alpha}^{h} \sum_{\beta \neq \alpha} \frac{G(\beta)-G(\alpha)}{|\beta-\alpha|} .
\end{aligned}
$$

After a similar interchange of the order of summation in the first term on the right-hand side of Eq. (A2), substitution of Eqs. (A2) and (A3) into Eq. (9) gives

$$
\begin{aligned}
\sum_{\alpha} G(\alpha) \mathbf{F}_{j, \alpha}^{h}= & -\gamma \sum_{\alpha} G(\alpha)\left[\mathbf{v}_{j, \alpha}-\mathbf{U}_{j, \alpha}\right]-\frac{3}{8}\left[\hat{\mathbf{I}}+\hat{\mathbf{u}}_{j} \hat{\mathbf{u}}_{j}\right] \\
& \cdot\left[\sum_{\alpha} G(\alpha) \mathbf{F}_{j, \alpha}^{h} \sum_{\beta \neq \alpha} \frac{1}{|\alpha-\beta|}+\Delta\right],
\end{aligned}
$$

where

$$
\Delta=\sum_{\alpha} \mathbf{F}_{j, \alpha}^{h} \sum_{\beta \neq \alpha} \frac{G(\beta)-G(\alpha)}{|\beta-\alpha|} .
$$

Consider the first contribution between the square brackets in Eq. (A4),

$$
\sum_{\alpha} G(\alpha) \mathbf{F}_{\alpha}^{h} \sum_{\beta \neq \alpha} \frac{1}{|\alpha-\beta|} .
$$

The sum $S(\alpha) \equiv \sum_{\beta \neq \alpha} 1 /|\alpha-\beta|$ can be approximated by an integral as before. To leading order one finds,

$$
\begin{aligned}
S(\alpha) & \equiv \sum_{\beta \neq \alpha} \frac{1}{|\alpha-\beta|} \\
& =\left[\int_{-(1 / 2)(L / D-1)}^{\alpha-1 / 2}+\int_{\alpha+1 / 2}^{(1 / 2)(L / D-1)}\right] d x \frac{1}{|x-\beta|} .
\end{aligned}
$$

The integrals are easily evaluated to yield

$$
\begin{aligned}
S(\alpha)= & 2 \ln \{2\}+\ln \left\{\frac{1}{2}\left(\frac{L}{D}-1\right)+\alpha\right\} \\
& +\ln \left\{\frac{1}{2}\left(\frac{L}{D}-1\right)-\alpha\right\} .
\end{aligned}
$$

Except for $\alpha$ 's close to the ends of the rod, this gives to leading order,

$$
S(\alpha) \approx 2 \ln \left\{\frac{L}{D}\right\} .
$$

In Fig. 2(a), $S(\alpha) / 2 \ln \{L / D\}$ is plotted as a function of $\alpha / m$, with $2 m+1$ the number of beads (so that $\alpha / m$ ranges from -1 to +1$)$. As can be seen, the approximation (A7) is good to within about $10 \%$, except at the very ends of the rod. In fact, the width of the region at the tips of the rod where Eq. (A7) is not a good approximation asymptotically vanishes in the limit where $L / D \rightarrow \infty$. Hence, except when $G(\alpha) \mathbf{F}_{j, \alpha}^{h}$ in Eq. (A6) peaks at the ends of rod $j$, Eq. (A7) can be used as a good approximation. For our purpose, there is no reason for the function $G(\alpha) \mathbf{F}_{j, \alpha}^{h}$ to peak at the very ends of the 

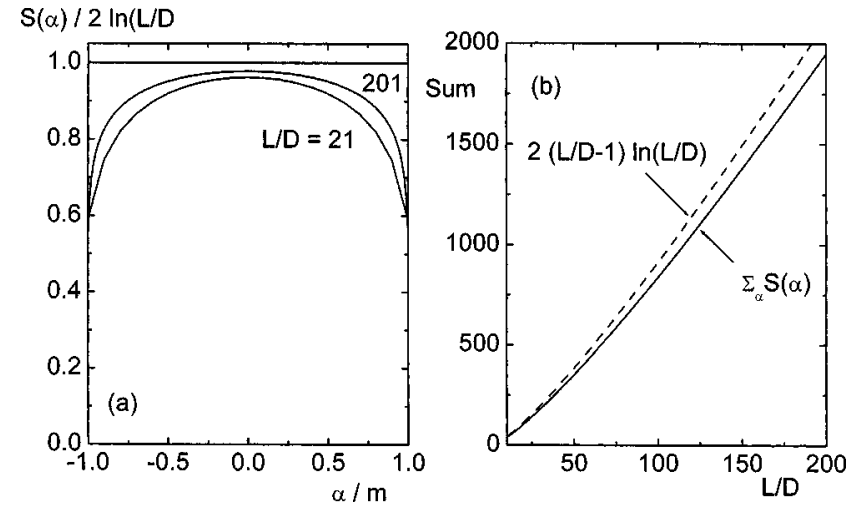

FIG. 2. (a) $S(\alpha) / 2 \ln \{L / D\}$ plotted as a function of $\alpha / m$, with $2 m+1$ the total number of beads (where $S(\alpha) \equiv \Sigma_{\beta \neq \alpha} 1 /|\alpha-\beta|$ ). The lower curve is for $L / D=20$, the upper curve for $L / D=201$. (b) The sums $\Sigma_{\alpha} S(\alpha)$ and $\Sigma_{\alpha}$ $2 \ln \{L / D\}=2(L / D-1) \ln \{L / D\}$ plotted as a function of $L / D$. The relative error between these two sums never exceeds $8 \%$, and very slowly converges to 0 with increasing $L / D$.

rod. A quantitative estimate for the error made in using Eq. (A7), is the difference between the sums $\Sigma_{\alpha} S(\alpha)$ and $\Sigma_{\alpha}$ $2 \ln \{L / D\}=2(L / D-1) \ln \{L / D\}$. These sums are plotted as functions of $L / D$ in Fig. 2(b). The relative error does not exceed $8 \%$ (for $L / D \leqslant 5$ ), and very slowly converges to 0 with increasing aspect ratio. Hence, to within about $10 \%$ error, we can approximate the expression in Eq. (A6) by

$$
\sum_{\alpha} G(\alpha) \mathbf{F}_{\alpha}^{h} \sum_{\beta \neq \alpha} \frac{1}{|\alpha-\beta|}=2 \ln \{L / D\} \sum_{\alpha} G(\alpha) \mathbf{F}_{j, \alpha}^{h} .
$$

The term on the left-hand side in Eq. (A4) can be neglected against this contribution, which is logarithmically larger. Next consider the contribution $\Delta$ in Eq. (A4), which is defined in Eq. (A5). There are three instances in the main text where the form (A5) is assumed to be very small in comparison to the first term in the square brackets in Eq. (A4), which is given to a good approximation by Eq. (A8): in calculating the total hydrodynamic force $\mathbf{F}_{j}^{h}$ in Eq. (11) to arrive at Eq. (15) for the translational velocity [in which case $G(\alpha)$ $=1]$, in calculating the total hydrodynamic torque $\mathbf{T}_{j}^{h}$ in Eq. (13) to arrive at Eq. (19) for the rotational velocity [in which case $G(\alpha)=\alpha$ ], and in going from Eq. (27) to Eq. (28) [for which case $G(\alpha)$ will be specified in the following].

For $G(\alpha)=1$, it trivially follows from Eq. (A5) that $\Delta$ $=0$. This immediately validates the use of Eq. (10) to arrive at Eq. (15) for the translational velocity.

Consider now the case where $G(\alpha)=\alpha$. From Eq. (A5) we have

$$
\Delta=\sum_{\alpha} \mathbf{F}_{j, \alpha}^{h}\left\{\sum_{\beta<\alpha}(-1)+\sum_{\beta>\alpha}(+1)\right\} .
$$

Since,

$$
\begin{aligned}
& \sum_{\beta<\alpha}(-1)=-\left\{\alpha+\frac{1}{2}\left(\frac{L}{D}-1\right)\right\}, \\
& \sum_{\beta>\alpha}(+1)=\left\{\frac{1}{2}\left(\frac{L}{D}-1\right)-\alpha\right\},
\end{aligned}
$$

we arrive at

$$
\Delta=-2 \sum_{\alpha} \alpha \mathbf{F}_{j, \alpha}^{h},
$$

which is of the order $1 / \ln \{L / D\}$ smaller relative to the expression in Eq. (A8). Hence, we can neglect the second term in square brackets in Eq. (A4) against the first term in case $G(\alpha)=\alpha$. This justifies the step required to arrive at Eq. (19) for the rotational velocity.

Finally, for the divergence of the stress tensor in Eq. (27) we need to evaluate the sum

$$
S \equiv \sum_{\alpha}\left\langle\delta\left(\mathbf{r}-\mathbf{r}_{1}-\alpha D \hat{\mathbf{u}}_{1}\right) \mathbf{F}_{1, \alpha}^{h}\right\rangle
$$

where $j$ is taken equal to 1 for convenience. Writing the ensemble average in terms of an integral with respect to the probability density function (pdf) $P$ of all phase space coordinates of the colloidal rods, the integration with respect to $\mathbf{r}_{1}$ can be done immediately due to the delta distribution, leading to

$$
\begin{aligned}
S= & \oint d \hat{\mathbf{u}}_{1} \int d \boldsymbol{\Gamma} \sum_{\alpha} P\left(\mathbf{r}_{1}=\mathbf{r}-\alpha D \hat{\mathbf{u}}_{1}, \hat{\mathbf{u}}_{1}, \boldsymbol{\Gamma}, t\right) \\
& \times \mathbf{F}_{1, \alpha}^{h}\left(\mathbf{r}_{1}=\mathbf{r}-\alpha D \hat{\mathbf{u}}_{1}, \hat{\mathbf{u}}_{1}, \boldsymbol{\Gamma}\right),
\end{aligned}
$$

where $\boldsymbol{\Gamma}$ stands for the phase space coordinates $\mathbf{r}_{2}, \cdots, \mathbf{r}_{N}, \hat{\mathbf{u}}_{2}, \cdots, \hat{\mathbf{u}}_{N}$. The integrand is of the form of the left hand-side of Eq. (9), except that in $\mathbf{F}_{1, \alpha}^{h}$ the position $\mathbf{r}_{1}$ is taken equal to $\mathbf{r}-\alpha D \hat{\mathbf{u}}_{1}$, which does not affect the present analysis leading to Eq. (10). The function $G(\alpha)$ is now equal to

$$
G(\alpha)=P\left(\mathbf{r}_{1}=\mathbf{r}-\alpha D \hat{\mathbf{u}}_{1}, \hat{\mathbf{u}}_{1}, \boldsymbol{\Gamma}, t\right) .
$$

Since the pdf is a continuous differentiable function of $\mathbf{r}_{1}$, there is a scalar $z$ between $\alpha$ and $\beta$, such that

$$
\frac{G(\beta)-G(\alpha)}{\beta-\alpha}=\frac{d G(z)}{d z} \equiv \frac{d P\left(\mathbf{r}_{1}=\mathbf{r}-z D \hat{\mathbf{u}}_{1}, \hat{\mathbf{u}}_{1}, \boldsymbol{\Gamma}, t\right)}{d z} .
$$

The latter derivative is just the change of $P$ on changing the position of rod number 1 by $D \hat{\mathbf{u}}_{1}$, that is, its center is shifted over a distance $D$ in the direction of its orientation. Since for the very large aspect ratios under consideration, suspension properties are essentially constant over distances of the order $D$, this is a very small number. The number $R$ $=(L / D) d \ln \{G(z)\} / d z$ measures the change of "the entropy" $\ln \{P\}$ over distances of the order of the length of the rods. In terms of this number we have the order of magnitude estimate,

$$
\Delta \sim R \sum_{\alpha} G(\alpha) \mathbf{F}_{1, \alpha}^{h} .
$$

Hence, according to Eq. (A8), as long as relative changes of suspension properties over the contour of the rods are much smaller than $\ln \{L / D\}, \Delta$ can be neglected against the first term between the square brackets in Eq. (A4). This justifies the step from Eq. (27) to Eq. (28). 


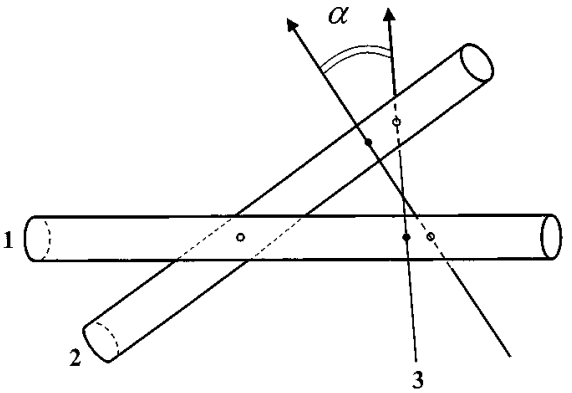

FIG. 3. The effective integration range of $\hat{\mathbf{u}}_{3}$ in the first-order density contribution to the pair-correlation function extends over the angle $\alpha$ which is at most of the order $D / L$

\section{APPENDIX B: DENSITY EXPANSION OF THE PAIR-CORRELATION FUNCTION}

The first two terms in the density expansion of the equilibrium pair-correlation function are

$$
\begin{aligned}
g\left(\mathbf{r}_{1}-\right. & \left.\mathbf{r}_{2}, \hat{\mathbf{u}}_{1}, \hat{\mathbf{u}}_{2}\right) \\
= & \exp \left\{V\left(\mathbf{r}_{1}-\mathbf{r}_{2}, \hat{\mathbf{u}}_{1}, \hat{\mathbf{u}}_{2}\right)\right\} \\
& \times\left\{1+\bar{\rho} \int d \mathbf{r}_{3} \oint d \hat{\mathbf{u}}_{3} \chi\left(\mathbf{r}_{1}-\mathbf{r}_{3}, \hat{\mathbf{u}}_{1}, \hat{\mathbf{u}}_{3}\right)\right. \\
& \left.\times \chi\left(\mathbf{r}_{3}-\mathbf{r}_{2}, \hat{\mathbf{u}}_{3}, \hat{\mathbf{u}}_{2}\right)+\cdots\right\} .
\end{aligned}
$$

Since the characteristic functions in the integrand are only nonzero when the core of rod number 3 overlaps with both the cores of rods 1 and 2, the integration with respect to $\hat{\mathbf{u}}_{3}$ effectively extends over an angular range of the order $D / L$, and the integration with respect to $\mathbf{r}_{3}$ contributes at most up to order $D L^{2}$ (see Fig. 3). Hence, the second term in the above-given expression is at most of order $(D / L) D L^{2} \bar{\rho}$ $\sim \varphi$. Since the volume fractions of interest scale like $D / L$, the first order in density contribution to the pair-correlation function is negligibly small for very long and thin rods. Higher order terms are similarly very small.

\section{APPENDIX C: AVERAGE DISTANCE BETWEEN BEADS}

The average distance $\bar{r}$ between beads on two distinct rods is measured by

$$
\begin{aligned}
\bar{r}^{2} & =\left(\frac{D}{L}\right)^{2} \sum_{\alpha_{1}, \alpha_{2}}\left\langle\left|\left(\mathbf{r}_{1}+\alpha_{1} \hat{\mathbf{u}}_{1} D\right)-\left(\mathbf{r}_{2}+\alpha_{2} \hat{\mathbf{u}}_{2} D\right)\right|^{2}\right\rangle \\
& =\left\langle\left|\mathbf{r}_{1}-\mathbf{r}_{2}\right|^{2}\right\rangle+\frac{D^{4}}{L^{2}} \sum_{\alpha_{1}, \alpha_{2}}\left(\alpha_{1}^{2}+\alpha_{2}^{2}\right),
\end{aligned}
$$

where $\mathbf{r}_{j}$ is the position coordinate of $\operatorname{rod} j$ and $\hat{\mathbf{u}}_{j}$ its orientation (see Fig. 1). Summations range over bead number indices, which are introduced at the beginning of Sec. III. Since $\left\langle\left|\mathbf{r}_{1}-\mathbf{r}_{2}\right|^{2}\right\rangle \sim \bar{\rho}^{-2 / 3}$, with $\bar{\rho}$ the particle number density, and the volume of a rod is $\sim D^{2} L$, it is found that

$$
\left\langle\left|\mathbf{r}_{1}-\mathbf{r}_{2}\right|^{2}\right\rangle \sim\left(\frac{L}{D}\right)^{4 / 3}\left(\frac{L}{D} \varphi\right)^{-2 / 3} D^{2} .
$$

Replacing sums by integrations (see Appendix B), which is allowed for long and thin rods, it is found that, $\Sigma_{\alpha} \alpha^{2}$ $=\frac{1}{12}(L / D)^{3}$, and hence,

$$
\sum_{\alpha_{1}, \alpha_{2}}\left(\alpha_{1}^{2}+\alpha_{2}^{2}\right)=\frac{1}{6}\left(\frac{L}{D}\right)^{4} .
$$

Since for the volume fractions of interest, $(L / D) \varphi$ is $\mathcal{O}(1)$, the leading contribution to $\bar{r}$ is thus given by

$$
\bar{r} / D \sim L / D .
$$

This result shows that the average distance between beads, measured in units of their own diameter, diverges as the aspect ratio diverges.

\section{APPENDIX D: DERIVATION OF EQ. (30)}

After substitution of Eqs. (14), (15), (19), and (29) into Eq. (28) it is immediately found that

$$
\nabla \cdot \boldsymbol{\Sigma}=\eta_{0} \nabla^{2} \mathbf{U}(\mathbf{r}, t)-\nabla P^{s s}(\mathbf{r}, t)+\mathbf{I}_{r}+\mathbf{I}_{t}+\mathbf{I}_{u},
$$

where

$$
\begin{aligned}
\mathbf{I}_{r}= & -\frac{4}{3} \frac{\gamma D D_{r}}{\ln \{L / D\}}\left(\sum_{j=1}^{N} \sum_{\alpha} \alpha \delta\left(\mathbf{r}-\mathbf{r}_{j}-\alpha D \hat{\mathbf{u}}_{j}\right)\right. \\
& \left.\times\left[\beta \hat{\mathcal{R}}_{j} \Psi+\hat{\mathcal{R}}_{j} \ln \{P\}\right] \times \hat{\mathbf{u}}_{j}\right), \\
\mathbf{I}_{t}= & -\frac{4}{3} \frac{\gamma D_{t}}{\ln \{L / D\}}\left(\sum_{j=1}^{N} \sum_{\alpha} \delta\left(\mathbf{r}-\mathbf{r}_{j}-\alpha D \hat{\mathbf{u}}_{j}\right)\right. \\
& \left.\times\left[\beta \nabla_{j} \Psi+\nabla_{j} \ln \{P\}\right]\right\rangle,
\end{aligned}
$$

and

$$
\begin{aligned}
\mathbf{I}_{u}= & \frac{4}{3} \frac{\gamma}{\ln \{L / D\}}\left\langle\sum_{j=1}^{N}\left[\hat{\mathbf{I}}-\frac{1}{2} \hat{\mathbf{u}}_{j} \hat{\mathbf{u}}_{j}\right]\right. \\
& \cdot \sum_{\alpha} \delta\left(\mathbf{r}-\mathbf{r}_{j}-\alpha D \hat{\mathbf{u}}_{j}\right) \\
& \times\left[\frac{D}{L} \sum_{\beta} \mathbf{U}_{j, \beta}-\mathbf{U}_{j, \alpha}-12 \alpha\left(\frac{D}{L}\right)^{3}\right. \\
& \left.\left.\times \hat{\mathbf{u}}_{j}\left\{\hat{\mathbf{u}}_{j} \times \sum_{\beta} \beta \mathbf{U}_{j, \beta}\right\}\right]\right) .
\end{aligned}
$$

First consider the relatively simple contribution

$$
\begin{aligned}
\mathbf{I} \equiv & \left\langle\sum_{j=1}^{N} \sum_{\alpha} \alpha \delta\left(\mathbf{r}-\mathbf{r}_{j}-\alpha D \hat{\mathbf{u}}_{j}\right) \hat{\mathbf{u}}_{j} \times \hat{\mathcal{R}}_{j} \ln \{P\}\right\rangle \\
= & \sum_{j=1}^{N} \sum_{\alpha} \alpha \int d \mathbf{r}_{1} \cdots \int d \mathbf{r}_{N} \oint d \hat{\mathbf{u}}_{1} \cdots \oint d \hat{\mathbf{u}}_{N} \\
& \times \delta\left(\mathbf{r}-\mathbf{r}_{j}-\alpha D \hat{\mathbf{u}}_{j}\right) \hat{\mathbf{u}}_{j} \times \hat{\mathcal{R}}_{j} P
\end{aligned}
$$

that appears in Eq. (D2) for $\mathbf{I}_{r}$. In the second line it is used that $P \hat{\mathcal{R}}_{j} \ln \{P\}=\hat{\mathcal{R}}_{j} P$, where, $P \equiv P\left(\mathbf{r}_{1}, \ldots, \hat{\mathbf{u}}_{N}, t\right)$ is the 
$N$-particle pdf. For each $j$, the integrations with respect to $\mathbf{r}_{m}$ and $\hat{\mathbf{u}}_{m}$ with $m \neq j$ can be done immediately. Assuming identical rods gives

$$
\begin{aligned}
\mathbf{I}= & N \sum_{\alpha} \alpha \int d \mathbf{r}_{1} \oint d \hat{\mathbf{u}}_{1} \delta\left(\mathbf{r}-\mathbf{r}_{1}-\alpha D \hat{\mathbf{u}}_{1}\right) \\
& \times \hat{\mathbf{u}}_{1} \times \hat{\mathcal{R}}_{1} P\left(\mathbf{r}_{1}, \hat{\mathbf{u}}_{1}, t\right) .
\end{aligned}
$$

It is to be noted that the differentiation with respect to $\hat{\mathbf{u}}_{1}$ must be performed, after which $\mathbf{r}_{1}$ can be replaced by $\mathbf{r}$ $-\alpha D \hat{\mathbf{u}}_{1}$ upon integration with respect to $\mathbf{r}_{1}$. Hence,

$$
\mathbf{I}=N \sum_{\alpha} \alpha \oint d \hat{\mathbf{u}}_{1} \hat{\mathbf{u}}_{1} \times\left[\hat{\mathcal{R}}_{1} P\left(\mathbf{r}-\alpha D \hat{\mathbf{u}}_{0}, \hat{\mathbf{u}}_{1}, t\right)\right] \hat{\mathbf{u}}_{0}=\hat{\mathbf{u}}_{1}
$$

Next, the summation over the bead index number $\alpha$ is replaced by an integral as

$$
\sum_{\alpha} f\left(\cdots-\alpha D \hat{\mathbf{u}}_{1}\right)=D^{-1} \int_{-L / 2}^{L / 2} d x f\left(\cdots-x \hat{\mathbf{u}}_{1}\right) .
$$

The corresponding contribution to Eq. (30) for the stress tensor is now found by using Eq. (21).

Next consider the somewhat more complicated contribution,

$$
\begin{aligned}
\mathbf{I} \equiv & \beta\left\langle\sum_{j=1}^{N} \sum_{\alpha} \alpha \delta\left(\mathbf{r}-\mathbf{r}_{j}-\alpha D \hat{\mathbf{u}}_{j}\right) \hat{\mathbf{u}}_{j} \times \hat{\mathcal{R}}_{j} \Psi\right\rangle \\
= & \beta \sum_{j=1}^{N} \sum_{\alpha} \alpha \int d \mathbf{r}_{1} \cdots \int d \mathbf{r}_{N} \oint d \hat{\mathbf{u}}_{1} \cdots \oint d \hat{\mathbf{u}}_{N} \\
& \times \delta\left(\mathbf{r}-\mathbf{r}_{j}-\alpha D \hat{\mathbf{u}}_{j}\right) P \hat{\mathbf{u}}_{j} \times \hat{\mathcal{R}}_{j} \Psi,
\end{aligned}
$$

which appears in Eq. (D2) for $\mathbf{I}_{r}$. Using pairwise additivity,

$$
\Psi\left(\mathbf{r}_{1}, \ldots, \mathbf{r}_{N}, \hat{\mathbf{u}}_{1}, \ldots, \hat{\mathbf{u}}_{N}\right)=\sum_{i<j} V\left(\mathbf{r}_{i}-\mathbf{r}_{j}, \hat{\mathbf{u}}_{i}, \hat{\mathbf{u}}_{j}\right)
$$

substitution of Eqs. (23), (25) together with Eq. (26), with $\nabla$ replaced by $\hat{\mathcal{R}}_{1}$, and assuming identical rods, it is readily found that

$$
\begin{aligned}
\mathbf{I}= & \sum_{\alpha} \alpha \int d \mathbf{r}_{1} \oint d \hat{\mathbf{u}}_{1} \oint d \hat{\mathbf{u}}_{2} \delta\left(\mathbf{r}-\mathbf{r}_{1}-\alpha D \hat{\mathbf{u}}_{1}\right) \rho\left(\mathbf{r}_{1}, \hat{\mathbf{u}}_{1}, t\right) \\
& \times \hat{\mathbf{u}}_{1} \times \hat{\mathcal{R}}_{1} \int d \mathbf{r}_{2} \rho\left(\mathbf{r}_{2}, \hat{\mathbf{u}}_{2}, t\right) \chi\left(\mathbf{r}_{1}-\mathbf{r}_{2}, \hat{\mathbf{u}}_{1}, \hat{\mathbf{u}}_{2}\right) .
\end{aligned}
$$

The integration with respect to $\mathbf{r}_{2}$ can be performed after transforming to the integration variable $\mathbf{R}=\mathbf{r}_{1}-\mathbf{r}_{2}$,

$$
\begin{aligned}
\int & d \mathbf{r}_{2} \rho\left(\mathbf{r}_{2}, \hat{\mathbf{u}}_{2}, t\right) \chi\left(\mathbf{r}_{1}-\mathbf{r}_{2}, \hat{\mathbf{u}}_{1}, \hat{\mathbf{u}}_{2}\right) \\
= & \int d \mathbf{R} \rho\left(\mathbf{r}_{1}-\mathbf{R}, \hat{\mathbf{u}}_{2}, t\right) \chi\left(\mathbf{R}, \hat{\mathbf{u}}_{1}, \hat{\mathbf{u}}_{2}\right) \\
= & 2 D\left|\hat{\mathbf{u}}_{1} \times \hat{\mathbf{u}}_{2}\right| \int_{-L / 2}^{L / 2} d l \int_{-L / 2}^{L / 2} d l^{\prime} \\
& \times \rho\left(\mathbf{r}_{1}-l \hat{\mathbf{u}}_{1}-l^{\prime} \hat{\mathbf{u}}_{2}, \hat{\mathbf{u}}_{2}, t\right) .
\end{aligned}
$$

In the second equation, the integration with respect to $\mathbf{R}$ is transformed to integration with respect to $\left\{l, l^{\prime}, l^{\prime \prime}\right\}$, which are defined as

$$
\begin{aligned}
& \mathbf{R}=l \hat{\mathbf{u}}_{1}+l^{\prime} \hat{\mathbf{u}}_{2}+l^{\prime \prime} \frac{\hat{\mathbf{u}}_{1} \times \hat{\mathbf{u}}_{2}}{\left|\hat{\mathbf{u}}_{1} \times \hat{\mathbf{u}}_{2}\right|}, \\
& -\frac{1}{2} L \leqslant l, l^{\prime} \leqslant \frac{1}{2} L, \quad-D \leqslant l^{\prime \prime} \leqslant D .
\end{aligned}
$$

The Jacobian of this transformation is equal to $\left|\hat{\mathbf{u}}_{1} \times \hat{\mathbf{u}}_{2}\right|$. Since the suspension properties do not significantly change over distances of the order of the thickness $D$ of the rods, the integration with respect to $l^{\prime \prime}$ gives rise to a prefactor $2 D$. Hence,

$$
\begin{aligned}
\mathbf{I}= & 2 D \sum_{\alpha} \alpha \int d \mathbf{r}_{1} \oint d \hat{\mathbf{u}}_{1} \oint d \hat{\mathbf{u}}_{2} \int_{-L / 2}^{L / 2} d l \int_{-L / 2}^{L / 2} d l^{\prime} \\
& \times \delta\left(\mathbf{r}-\mathbf{r}_{1}-\alpha D \hat{\mathbf{u}}_{1}\right) \rho\left(\mathbf{r}_{1}, \hat{\mathbf{u}}_{1}, t\right) \\
& \times \hat{\mathbf{u}}_{1} \times \hat{\mathcal{R}}_{1}\left|\hat{\mathbf{u}}_{1} \times \hat{\mathbf{u}}_{2}\right| \rho\left(\mathbf{r}_{1}-l \hat{\mathbf{u}}_{1}-l^{\prime} \hat{\mathbf{u}}_{2}, \hat{\mathbf{u}}_{2}, t\right) .
\end{aligned}
$$

As before it should be noted that upon integration with respect to $\mathbf{r}_{1}$, the delta distribution renders $\mathbf{r}_{1}=\mathbf{r}-\alpha D \hat{\mathbf{u}}_{1}$ after the differentiation with respect to $\hat{\mathbf{u}}_{1}$ has been performed. Hence,

$$
\begin{aligned}
\mathbf{I}= & 2 D \sum_{\alpha} \alpha \oint d \hat{\mathbf{u}}_{1} \oint d \hat{\mathbf{u}}_{2} \int_{-L / 2}^{L / 2} d l \int_{-L / 2}^{L / 2} d l^{\prime} \\
& \times \rho\left(\mathbf{r}-\alpha D \hat{\mathbf{u}}_{1}, \hat{\mathbf{u}}_{1}, t\right) \hat{\mathbf{u}}_{1} \times\left[\hat{\mathcal{R}}_{1}\left|\hat{\mathbf{u}}_{1} \times \hat{\mathbf{u}}_{2}\right|\right. \\
& \left.\rho\left(\mathbf{r}-\alpha D \hat{\mathbf{u}}_{0}-l \hat{\mathbf{u}}_{1}-l^{\prime} \hat{\mathbf{u}}_{2}, \hat{\mathbf{u}}_{2}, t\right)\right] \hat{\mathbf{u}}_{0}=\hat{\mathbf{u}} .
\end{aligned}
$$

The bead index summation is replaced by an integral similarly as in Eq. (D5), leading to (with $\hat{\mathbf{u}}=\hat{\mathbf{u}}_{1}$ and $\hat{\mathbf{u}}^{\prime}=\hat{\mathbf{u}}_{2}$ )

$$
\begin{aligned}
\mathbf{I}= & \frac{2}{D} \oint d \hat{\mathbf{u}} \oint d \hat{\mathbf{u}}^{\prime} \int_{-L / 2}^{L / 2} d x \int_{-L / 2}^{L / 2} d l \int_{-L / 2}^{l / 2} d l^{\prime} x \\
& \times \rho(\mathbf{r}-x, \hat{\mathbf{u}}, t) \hat{\mathbf{u}} \times\left[\hat{\mathcal{R}}\left|\hat{\mathbf{u}} \times \hat{\mathbf{u}}^{\prime}\right|\right. \\
& \left.\rho\left(\mathbf{r}-x \hat{\mathbf{u}}_{0}-l \hat{\mathbf{u}}-l^{\prime} \hat{\mathbf{u}}^{\prime}, \hat{\mathbf{u}}, t\right)\right]_{\hat{\mathbf{u}}_{0}=\hat{\mathbf{u}}} .
\end{aligned}
$$

This expression can be found in Eq. (30).

The contribution $\mathbf{I}_{t}$ to the stress tensor in Eq. (D3) is evaluated similarly.

The $\beta$ summations in the contribution $\mathbf{I}_{u}$ in Eq. (D4) are replaced by integrals, similar to Eq. (D5), as

$$
\sum_{\beta} \beta \mathbf{U}_{j, \beta}=D^{-2} \int d x^{\prime} x^{\prime} \mathbf{U}\left(\mathbf{r}_{j}+x^{\prime} \hat{\mathbf{u}}_{j}\right) .
$$


The prefactors in Eqs. (D2)-(D4) are found from Eqs. (16) and (20) to be equal to

$$
\frac{4}{3} \frac{\gamma D_{t}}{\ln \{L / D\}}=\frac{D}{L} k_{B} T, \quad \frac{4}{3} \frac{\gamma D D_{r}}{\ln \{L / D\}}=12 \frac{D^{2}}{L^{3}} k_{B} T .
$$

This concludes the mathematical details on the explicit evaluation of the stress tensor leading to Eq. (30).

${ }^{1}$ Faraday Discuss. (to be published).

${ }^{2}$ V. Schmitt, F. Lequeux, A. Pousse, and D. Roux, Langmuir 10, 955 (1994).

${ }^{3}$ J.-F. Berret, D. Roux, and G. Porte, J. Phys. II 4, 1261 (1994).

${ }^{4}$ P. T. Callaghan, M. E. Cates, C. J. Rofe, and J. B. A. F. Smeulders, J. Phys. II 6, 375 (1996).

${ }^{5}$ P. Boltenhagen, Y. Hu, E. F. Matthys, and D. J. Pine, Phys. Rev. Lett. 79, 2359 (1997).

${ }^{6}$ O. Diat, D. Roux, and F. Nallet, J. Phys. II 3, 1427 (1993).

${ }^{7}$ D. Roux, F. Nallet, and O. Diat, Europhys. Lett. 24, 53 (1993).

${ }^{8}$ A. Imhof, A. van Blaaderen, and J. K. G. Dhont, Langmuir 10, 3477 (1994).

${ }^{9}$ T. Palberg and M. Würth, J. Phys. I 6, 237 (1996).

${ }^{10}$ D. Bonn, J. Meunier, O. Greffier, A. Al-Kahwaij, and H. Kellay, Phys. Rev. E 58, 2115 (1998).

${ }^{11}$ P. T. Callaghan, Rep. Prog. Phys. 62, 599 (1999).
${ }^{12}$ J. Vermant, J. Colloid Interface Sci. 211, 221 (1999).

${ }^{13}$ T. A. J. Lenstra, Z. Dogic, and J. K. G. Dhont, J. Chem. Phys. 114, 10151 (2001).

${ }^{14}$ P. D. Olmsted and P. M. Goldhart, Phys. Rev. A 46, 4966 (1992).

${ }^{15}$ P. D. Olmsted and C.-Y. D. Lu, Phys. Rev. E 56, 55 (1997).

${ }^{16}$ P. D. Olmsted and C.-Y. D. Lu, Faraday Discuss. 112, 1 (1999).

${ }^{17}$ J. K. G. Dhont and W. J. Briels (unpublished).

${ }^{18}$ J. K. G. Dhont and W. J. Briels, J. Chem. Phys. 117, 3992 (2002).

${ }^{19}$ G. K. Batchelor, J. Fluid Mech. 41, 545 (1970); 83, 97 (1977).

${ }^{20}$ M. Doi and S. F. Edwards, The Theory of Polymer Dynamics (Clarendon, Oxford, 1986).

${ }^{21}$ S. Kim and S. J. Karrila, Hydrodynamics: Principles and Selected Applications (Butterworth-Heinemann, Boston, 1991).

${ }^{22}$ J. K. G. Dhont, An Introduction to Dynamics of Colloids (Elsevier, Amsterdam, 1996).

${ }^{23}$ The orientationaly averaged diffusion tensor $\left\langle D_{t}[\hat{\mathbf{I}}+\hat{\mathbf{u}} \hat{\mathbf{u}}]\right\rangle$ is equal to $\hat{\mathbf{I}} \bar{D}$, where $\bar{D}=\frac{4}{3} D_{t}$ is more commonly referred to as "the translational diffusion coefficient" of a rod. Here we shall refer to $D_{t}$ in Eq. (16) as the translational diffusion coefficient.

${ }^{24}$ L. Onsager, Chem. Rev. 13, 73 (1933); Phys. Rev. 62, 558 (1942); Ann. N.Y. Acad. Sci. 51, 627 (1949).

${ }^{25}$ P. van der Schoot and T. Odijk, J. Chem. Phys. 97, 515 (1992).

${ }^{26}$ J. K. G. Dhont and W. J. Briels (unpublished).

${ }^{27}$ G. Marrucci and F. Greco, Advances in Chemical Physics, edited by I. Prigogine and S. A. Rice (Wiley, New York, 1993), Vol. LXXXVI. 